\title{
Optimization of Unmanned Aerial Vehicles: Expanding the Multidisciplinary Capabilities
}

\author{
Athanasios Papageorgiou
}

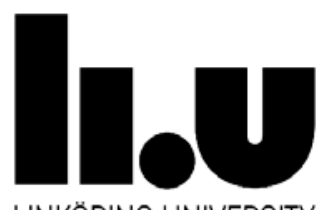

LINKÖPING UNIVERSITY

Division of Machine Design

Department of Management and Engineering Linköping University, SE-581 83 Linköping, Sweden 
Copyright (C) Athanasios Papageorgiou, 2017

Optimization of Unmanned Aerial Vehicles: Expanding the Multidisciplinary Capabilities

ISBN: 978-91-7685-391-7

ISSN 0280-7971

Distributed by:

Division of Machine Design

Department of Management and Engineering

Linköping University

SE-581 83 Linköping, Sweden

Printed in Sweden by LiU-Tryck, Linköping, 2017 
To my Friends and Family 


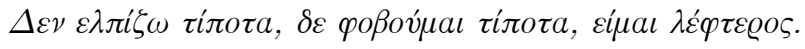

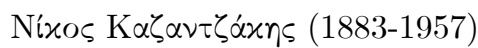

I hope for nothing, I fear nothing, I am free.

Nikos Kazantzakis (1883-1957) 


\section{Abstract}

Over the last decade, Unmanned Aerial Vehicles (UAVs) have experienced an accelerated growth, and nowadays they are being deployed in a variety of missions that have traditionally been covered by manned aircraft. This unprecedented market expansion has created new and unforeseen challenges for the manufacturing industry which is now called to further reduce the idea-to-market times while simultaneously delivering designs of even higher performance. In this environment of uncertainty and risk, it is without a doubt crucial for the involved actors to find ways to secure their strategic advantage, and hence, implementing the latest design tools has become a critical consideration in every Product Development Process (PDP).

To this end, a method that has been frequently applied in the PDP and has shown many successful results in the development of complex engineering products is Multidisciplinary Design Optimization (MDO). In general, MDO can bring additional knowledge regarding the best-suited designs much earlier in the process, and in this respect, it can lead to significant cost and time savings by reducing the total number of refinement iterations. Nevertheless, the organizational and cultural integration of MDO has been often overlooked, while at the same time, several technical aspects of the method for UAV design are still at an elementary level. On the whole, research on MDO is showing a slow progress, and to this date, there are many limitations in both the disciplinary models and the available analysis capabilities.

In light of the above, this thesis focuses on the particulars of the MDO methodology, and more specifically, on how it can be best adapted and evolved in order to enhance the development process of UAVs. The primary objective is to study the current trends and gaps of the MDO practices in UAV applications, and subsequently to build upon that and explore how these can be included in a roadmap that will be able to serve a guide for newcomers in the field. Compared to other studies, the problem is herein approached from both a technical as well as organizational perspective, and thus, this research not only aims to propose techniques that can lead to better designs but also solutions that will be meaningful to the PDP. Having established the above foundation, this work shows that the traditional MDO frameworks for UAV design have been neglecting several important features, and it elaborates on how those novel elements can be modeled in order to enable a better integration of MDO into the organizational functions. Overall, this thesis presents quantitative and qualitative data which illustrate the effectiveness of the new framework enhancements in the development process of UAVs, and concludes with discussions on the possible improvement directions towards achieving more and better MDO capabilities. 


\section{Acknowledgements}

The research work that is presented in this thesis has been conducted at the Division of Machine Design of Linköping University and it was funded by the Innovative Multidisciplinary Product Optimization (IMPOz) project which was managed by the Swedish innovation agency VINNOVA.

First and foremost, I would like to thank my supervisor Professor Johan Ölvander for believing in me and for giving me the opportunity to work in his research group. Thank you for introducing me to the wonderful field of engineering design optimization and thank you for always being there to guide me with your valuable comments, thoughts, and ideas.

Furthermore, my special thanks goes to my two co-supervisors Dr. Mehdi Tarkian and Dr. Kristian Amadori for supporting me throughout this project and for continuously providing me with insightful advice and suggestions. Without a doubt, your previous work has been a source of true inspiration, and I cannot be grateful enough for all the commitment and trust that you have shown me since the beginning of this endeavor.

At this point, I would like to acknowledge the contribution of Dr. Christopher Jouannet from Saab Aeronautics who has been acting a link to the industry, despite his already busy schedule. Your technical insights have been undeniably vital towards the completion of this project, while your belief in my abilities has always been a very strong motivation.

My greatest appreciation goes also to my colleagues from the division of Machine Design and the division of Fluid and Mechatronic Systems for integrating me into their social activities. Thank you for all your administrative support with the everyday challenges and for all your efforts to create a pleasant working environment.

Last but not least, I would like to express my gratitude to my family and especially to my parents Giorgos and Pinelopi who have supported me unconditionally since the beginning of my studies in Sweden. You have helped my like no other person in my hour of need and you have taught me important values that should be the essence of every researcher and educator. I dedicate this thesis to you.

Linköping, December 2017

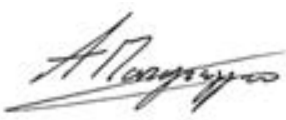

Athanasios Papageorgiou 


\section{Appended Papers}

The papers which are presented here constitute the research foundation of this work and have been appended to their full extent at the end of this thesis. In the following pages they will be referred to by using the Roman enumeration which is seen below:

[I] Papageorgiou A., Tarkian M., Amadori K., and Ölvander J., "Review of Multidisciplinary Optimization Practices: A Roadmap for Unmanned Aerial Vehicle Design", Submitted for journal publication, 2017

[II] Papageorgiou A., and Ölvander J., "The role of multidisciplinary design optimization (MDO) in the development process of complex engineering products", Proceedings of the 21st International Conference on Engineering Design (ICED 17), Vancouver, Canada, 2017

[III] Papageorgiou A., Ölvander J., and Amadori K., "Development of a Multidisciplinary Design Optimization Framework Applied on UAV Design by Considering Models for Mission, Surveillance, and Stealth Performance", Proceedings of the 18th AIAA/ISSMO Multidisciplinary Analysis and Optimization Conference, Denver, Colorado, 2017

[IV] Papageorgiou A., Tarkian M., Amadori K., and Ölvander J., "Multidisciplinary Optimization of Unmanned Aircraft Considering Radar Signature, Sensors, and Trajectory Constraints", Reviewed and accepted for publication in the AIAA Journal of Aircraft, 2017

In all the above papers, Papageorgiou is the main author and the main contributor. More specifically, in papers I and II, Papageorgiou carried out the literature review, performed the analysis of the findings, and wrote the manuscript. Accordingly, in papers III and IV, Papageorgiou developed the models but also the framework, carried out the optimization as well as the analysis of the results, and wrote the manuscript. The co-authors that are listed in the above papers provided feedback. 


\section{Abbreviations}

$\begin{array}{ll}\text { AAO } & \text { All-At-Once } \\ \text { AKR } & \text { Anisotropic Kriging } \\ \text { CAD } & \text { Computer Aided Design } \\ \text { CFD } & \text { Computational Fluid Dynamics } \\ \text { CO } & \text { Collaborative Optimization } \\ \text { CSM } & \text { Computational Structural Mechanics } \\ \text { DOE } & \text { Design Of Experiments } \\ \text { DOF } & \text { Degrees Of Freedom } \\ \text { DRM } & \text { Design Research Methodology } \\ \text { GA } & \text { Genetic Algorithms } \\ \text { GUI } & \text { Graphical User Interface } \\ \text { IDF } & \text { Individual Discipline Feasible } \\ \text { MDF } & \text { Multi-Disciplinary Feasible } \\ \text { MDO } & \text { Multidisciplinary Design Optimization } \\ \text { MOO } & \text { Multi-Objective Optimization } \\ \text { MTOW } & \text { Maximum Takeoff Weight } \\ \text { NN } & \text { Neural Networks } \\ \text { OML } & \text { Outer Mold Line } \\ \text { PDP } & \text { Product Development Process } \\ \text { PO } & \text { Physical Optics } \\ \text { RANS } & \text { Reynolds-Averaged Navier-Stokes } \\ \text { RCS } & \text { Radar Cross Section } \\ \text { SFC } & \text { Specific Fuel Consumption } \\ \text { SOM } & \text { Self-Organizing Maps } \\ \text { SOO } & \text { Single-Objective Optimization } \\ \text { UAV } & \text { Unmanned Aerial Vehicle } \\ \text { ULH } & \text { Uniform Latin Hypercube } \\ & \end{array}$




\section{Contents}

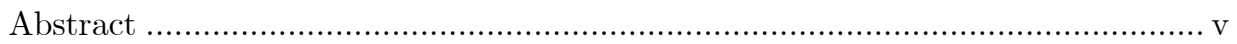

Acknowledgements ......................................................................... vii

Appended Papers ................................................................................. ix

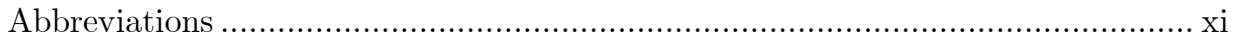

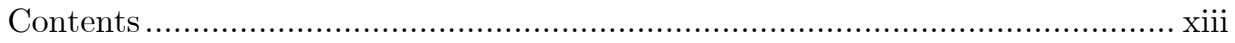

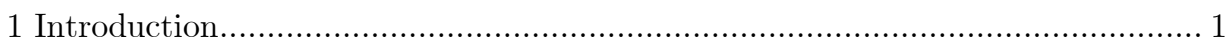

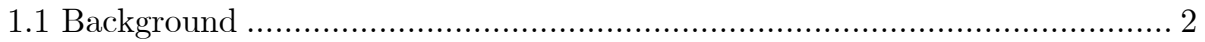

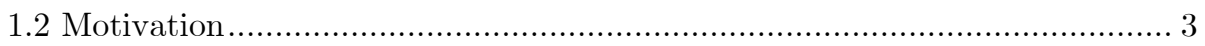

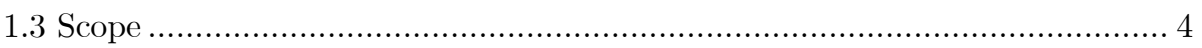

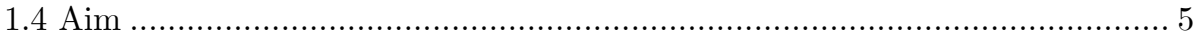

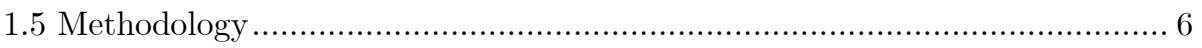

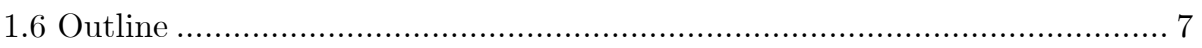

2 Identifying the Current Possibilities in MDO of UAVs ................................ 9

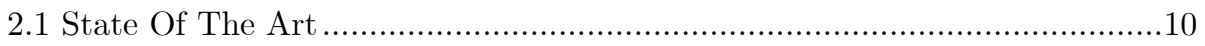

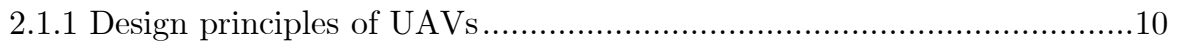

2.1.2 Engineering design optimization ............................................. 11

2.1.3 Decomposition of MDO problems ................................................13

2.1.4 Efficient computing methods ...................................................... 15

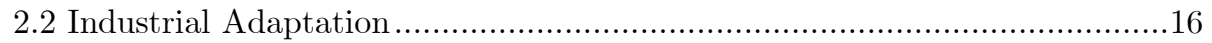

2.2.1 Enhancing the development process .............................................16

2.2.2 Achieving organizational integration .......................................... 17

2.2.3 Managing complex systems .................................................... 18

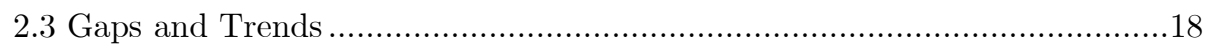

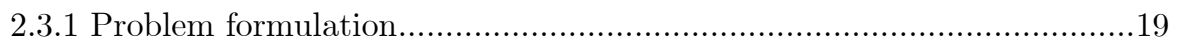

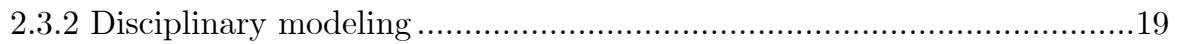

2.3.3 Analysis capabilities ...............................................................20

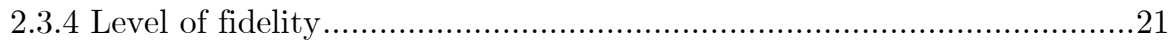


2.4 Improvement Directions ...................................................................22

2.4.1 General research assessment ..................................................22

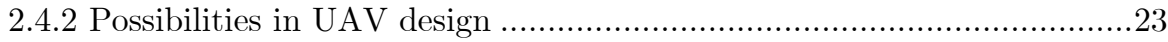

3 Expanding the Existing Capabilities in MDO of UAVs................................25

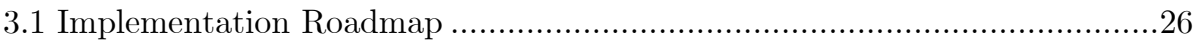

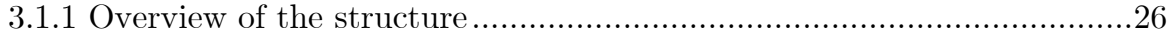

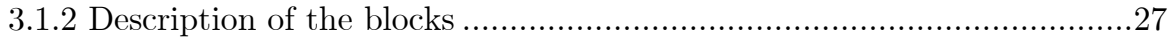

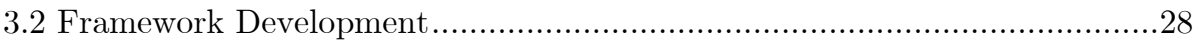

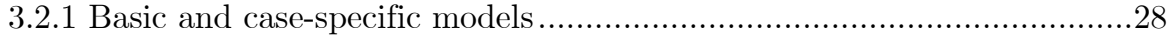

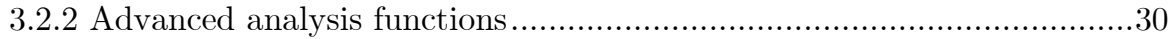

3.3 Computational Performance .................................................................

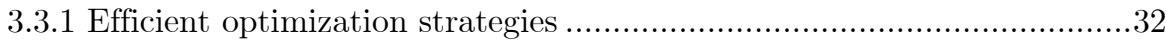

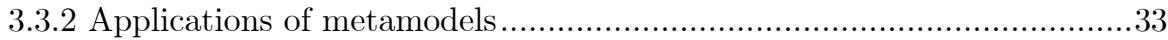

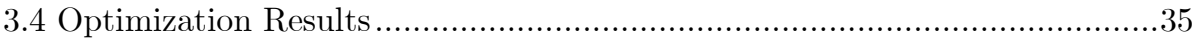

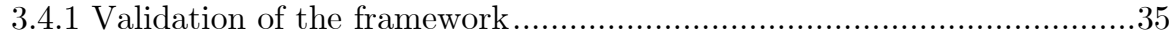

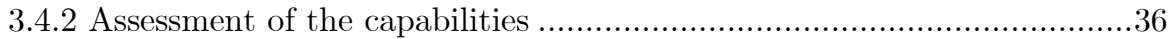

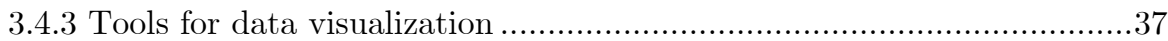

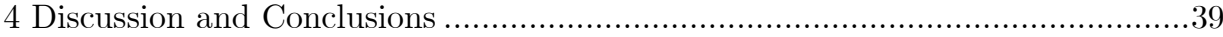

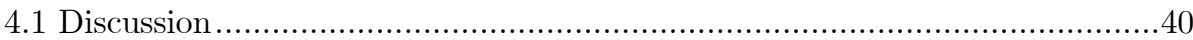

4.1.1 Current practices and future possibilities ......................................40

4.1.2 Improvement of the MDO capabilities ...........................................41

4.1.3 Enhancement of the development process .......................................42

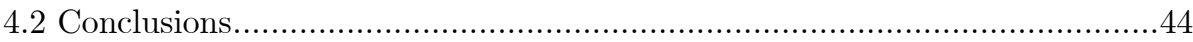

4.2.1 Answers to the research questions ................................................44

4.2.2 Outlook and future work .............................................................46

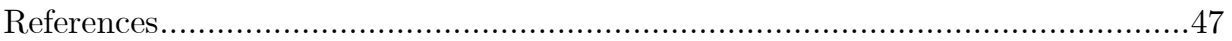




\section{1 \\ Introduction}

When aerial missions become too "dull, dirty, or dangerous" for humans, then the implementation of Unmanned Aerial Vehicles (UAVs) can be an indisputably advantageous alternative which can lead to considerable benefits both in terms of money but also time (Tice, 1991). In the past few years, the use of UAVs has experienced an accelerated and unprecedented growth, and at present, there is a wide range of applications that can be performed much safer and with less cost if manned operations can be avoided (Volpe, 2013). As expected, this competitive market is imposing more strict performance and delivery requirements, and as a direct consequence, companies are nowadays faced with new challenges that in turn call for even more efficient development tools.

Multidisciplinary Design Optimization (MDO) is a promising method that can be applied in the development process of complex products in order to explore the design tradeoffs by using analysis or numerical optimization. Nonetheless, MDO comes with several limitations, and it can be seen that the computational efficiency can often be the bottleneck when it comes to its implementation in the preliminary and detailed design applications. At the same time, there are still gaps in the disciplinary modeling and analysis capabilities, while a further and rather critical shortcoming is the lack of research regarding the integration of MDO within the organizational functions.

This thesis aims to explore the state-of-the-art methods for applying MDO on UAVs and in turn to draw a roadmap for guiding practitioners and newcomers into the field. The proposed roadmap focuses on the technical requirements and techniques that are currently used to enable efficient MDO, but it also emphasizes on the organizational aspects that need to be taken into account in such endeavors. Having established the aforementioned roadmap, this research investigates methods for improving the current gaps, while finally, as a step further it attempts to expand the traditional framework towards totally new, but yet essential, features for MDO of UAVs.

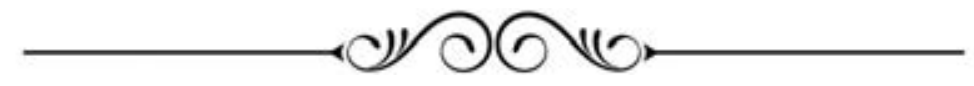




\subsection{Background}

The development of complex engineering systems is a multidisciplinary process which goes through many design phases and requires a significant amount of coordination between experts in order to ensure the success of the final product. In its simplest form, the Product Development Process (PDP) has a structure which resembles a stage-gate system, and it is usually comprised of several activities as well as checkpoints which control that the initial design requirements have been met (Cooper, 1990). The PDP starts from an abstract idea and ends with manufacturing, while during this time, it is typically necessary to bring several departments of the organization together so that they can identify a configuration that is holistically acceptable. To no surprise, the final outlook of the PDP will ultimately depend on the product specifications as well as the adopted practices of each company, and to this date, engineers can choose from a large selection of available tools which aim to enhance the design and accelerate the overall process (Cooper, 2014).

Although the PDP is comprised of many different segments that call for a wide range of domain-specific work, it can be argued that the core engineering activities take place during three key stages which are namely the conceptual, the preliminary, and the detailed design phases (Ulrich and Eppinger, 2012). The main problem here is that at the beginning of the process there is a lot of freedom to make choices, but on the other hand there is not so much knowledge on how the design will eventually perform. Conversely, in the later and more refined stages the design may be better understood, but at the same time, it has become very difficult to change it because this would require a significant amount of time but also company resources. This is especially critical in the development of complex engineering products with many underlying dependencies since it is often difficult to fully understand the complete system behavior unless there is sufficient data from multidisciplinary but also highfidelity simulations (Haskins et al., 2006). Overall, the above paradox shows that adequate knowledge of the design is a concept of utmost importance within the PDP, and in this respect, it is crucial to strive for more and better information even from the early stages of process (see Figure 1).

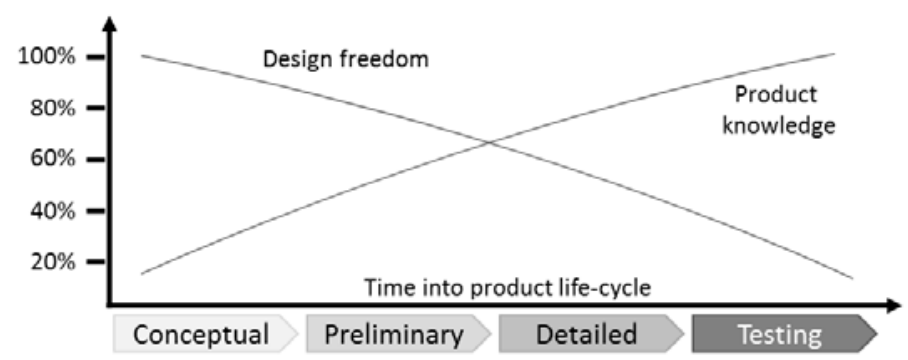

Figure 1. The paradox of design freedom against product knowledge, adapted from Karniel and Reich (2011). 
Given the above premise, it can be seen that the traditional PDP may be wellfitted for simple or existing products, but it often demonstrates several limitations in the development of complex systems like UAVs where there is typically a great deal of innovation as well as numerous components with intricate synergies (Crawley et al., 2004). To this end, MDO is able to take into account multiple disciplines at the same time, and hence, it is possible to consider several aspects of the design simultaneously instead of working on them in isolation as it has been commonly done in the conventional PDP. This leads to a more holistic view of the system interactions as well as a reduction of the costly iterations between the engineering teams, while depending on the fidelity of the tools, it can also allow for high-detail and thus more accurate design data to flow into the conceptual design stage (Agte et al., 2009). On the whole, the field of MDO has been constantly growing, and nowadays it is possible to enhance the speed and quality of the calculations by taking into account state-of-the-art analysis capabilities, advanced decomposition architectures, smart integration tools, and more efficient computing techniques (Simpson and Martins, 2011).

\subsection{Motivation}

One of the main objectives of the manufacturing industry is, and has always been, to be able to market successful products which can generate higher profits and in turn expand the market share of the organization. Offering better solutions compared to the competition is without doubt a critical factor for achieving a strategic advantage, and therefore, one key attribute towards the economic success of the company is to consider design tools that enable high performance designs but also faster and more agile processes. Although this is true for most products, it can be said that it becomes particularly critical in the development of UAVs where it can be seen that the market is galloping and there are currently very high demands for more quality as well as shorter delivery times (Volpe, 2013).

In this light, the main motivation for this work is to improve the PDP of UAVs with more state-of-the-art tools that will allow the design teams to take more supported decisions earlier in the process when there is still time to make the necessary alterations (Karniel and Reich, 2011). To accomplish this, it is first and foremost important to address the issue of allowing more design knowledge to become available as early as possible but also the issue of the costly and time-consuming iterations which occur due to the isolation of the engineering teams. In this respect, MDO appears as a promising design tool since it has the potential to provide more and better information at all stages of the PDP, while at the same time, it is also particularly suitable for UAVs since this type of product includes by nature multiple disciplines and sub-systems.

Even though MDO has been applied in several UAV development studies, it can be argued that there are still several gaps which limit the knowledge that this method 
can deliver and impede its eventual use within the PDP (Agte et al., 2009). More specifically, the majority of cases are only concentrating on conceptual design, whereas most of them have also been neglecting important design requirements by focusing solely on the aeronautical aspects of the aircraft. At the same time, the studies with high-fidelity tools, and thus more industrial interest, are reporting many issues regarding the computational efficiency of MDO, and to this date, this as well as other integrational limitations have evolved as a major hinder towards the complete implementation of MDO within the PDP (Simpson and Martins, 2011). Finally, most publications on MDO have been excessively focused on proving the technical benefits of its implementation, and as a result, there are currently many case studies on how to get optimized designs, but hardly any research on how those can be meaningfully used by the manufacturing industry (Belie, 2002).

\subsection{Scope}

The scope of this thesis is the enhancement of the PDP of UAVs through the use of MDO, and therefore, the research that is presented here is exclusively centered on these strictly defined research topics (see Figure 2).

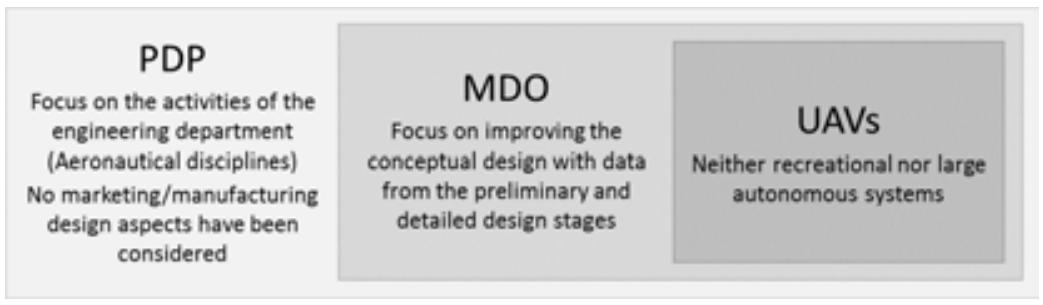

Figure 2. The scope and research topics that are covered by this thesis.

Given that UAVs share a large number of common elements with manned aircraft, it is herein considered relevant to take that into account, and hence, supplementary knowledge from this field is used when this is needed to improve or complement the contributions. Although the term "UAV" may refer to a very large spectrum of products, the focus of this research is on applications with a sufficient level of system complexity which suggests that neither the small-scale (recreational) applications nor the large autonomous systems have been included in this study.

In this context, MDO is approached more as an optimization method rather than a design space exploration strategy. The multidisciplinary nature is expressed through the simultaneous use of both disciplinary models and analysis capabilities under a common framework, and for this initial application the considered tools include basic aeronautical disciplines but also case-specific analytical functions in order to be able to capture the requirements of each optimization scenario. On the whole, the MDO in this thesis is about merging the conceptual and preliminary design stages, and as such, 
a multi-fidelity approach where data from high-fidelity analyses are brought into the conceptual design is herein employed.

As far as the PDP is concerned, it can be argued that the discussions which are presented in this study have only emphasized on the enhancement of the three main design stages (i.e. conceptual, preliminary, and detailed), whereas the front and back end of the process have been excluded. Overall, this research is about the additions that can bridge the above stages by increasing the knowledge early on in the process, and to this end, aspects such as planning, testing, and production have been intentionally left outside the scope of this work. Accordingly, the proposed enhancement methods have only targeted the activities and teams within the engineering department, and therefore, this thesis has neither considered nor tested a potential expansion towards other sections of the organization like for example marketing and manufacturing.

\subsection{Aim}

The aim of the present work is to provide a summary of the MDO field and in turn to identify the gaps and trends as well as the organizational effects of using optimization in UAV companies. Hence, the research has been performed from both an MDO and PDP perspective, and it has been collectively presented in the form of a roadmap that will serve as guide for both the academia and the industry. As a unique contribution, the implementation of novel elements to the traditional framework is herein investigated, and a framework for UAV design is subsequently developed by taking into account additional disciplinary models, new analysis capabilities, and more efficient computing techniques. Finally, the thesis concludes with a presentation of the obtained optimization results, and it is shown that adding "more of the same" together with "entirely new" features can be an ideal direction for improving the current methods for applying MDO on UAVs.

In total, the principle idea is the application of MDO in the PDP of UAVs and how this can be improved in order to enable better designs that will allow the organization to maximize its success. Given this foundation, this thesis collectively summarizes and discusses the results from all the appended papers, and as step further, it attempts to provide answers to three specific topics which are presented below in the form of research questions:

- RQ1: What are the current research gaps, trends, and improvement possibilities in $M D O$ of $U A V s$ ?

- RQ2: Which are the most critical additions towards enhancing the MDO of surveillance UAVs?

- RQ3: How can MDO be integrated in the manufacturing industry and support the PDP of $U A V s$ ? 


\subsection{Methodology}

The research methodology which was used throughout this work is in accordance with the Design Research Methodology (DRM) which was suggested by Blessing and Chakrabarti (2009). DRM has been specifically developed in order to fit studies in the field of engineering design, and it is comprised of four main stages which are typically addressed in an iterative way until the desired result has been achieved. A graphical representation of the DRM is given in Figure 3, while the four main stages are further elaborated below:

- Criteria: Identification of the aim and focus that the research is expected to fulfil and definition of a criterion that will act as a metric of success.

- Descriptive study 1: Use of literature and observations to understand the factors that influence the success and investigation of a foundation that will allow further work.

- Prescriptive study: Implementation of experience and assumptions in order to develop new methods and tools that will enable an improvement of the existing state of the art.

- Descriptive study 2: Evaluation of the effect that the proposed methods and tools have on the previously defined criterion of success and initiation of improvement iterations.

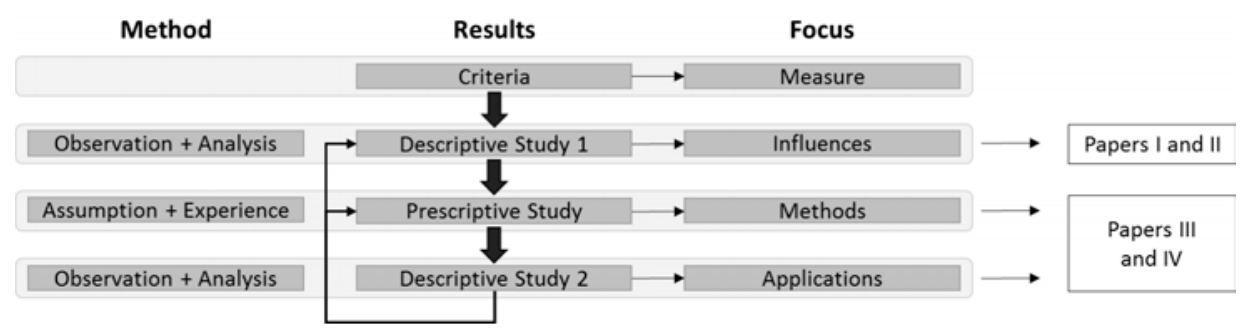

Figure 3. The Design Research Methodology (DRM) framework, adapted from Blessing and Chakrabarti (2009).

The first part, and hence the foundation of this work, are two literature reviews which aim to present the current research gaps and trends in MDO of UAVs, and subsequently to help identify the possible directions for improving the existing practices. By using this descriptive basis, the second part of this thesis is about the development of an MDO framework with more powerful capabilities, and in particular, about adding novel but also more efficient elements in order to better capture the performance of UAVs. Given this contribution, the last part of this study presents quantitative results that were obtained from two exploratory case studies, and concludes with an evaluation of the new additions by taking into account both the achieved design quality and the enhancement of the process. 


\subsection{Outline}

This research is for the most part based on the work that is presented in the appended papers, and therefore, it has been structured as a compilation thesis where information is only repeated when it is necessary to introduce a concept or keep the consistency of the text. Overall, this work is comprised of five chapters with the introduction being the first, then followed by the two main research topics, and finally summarizing with discussions and conclusions (see Figure 4). To avoid repetition, and given the fact that two of the appended papers are literature reviews, the theory that is presented in this thesis has been largely based on papers I and II, while some additional theoretical elements have also been added in order to provide the reader with a more holistic view of the field. Since papers I and II are a combination of stateof-the-art information and own research, chapter 2 includes both a theoretical background as well as contributions which are presented here in the form of identifying the current MDO possibilities. Therefore, chapter 2 should be viewed in this context as both a "theory" and a "contribution" chapter, while accordingly, further contributions regarding the expansion of the existing MDO capabilities are subsequently presented in chapter 3 which is based on papers I, III and IV.

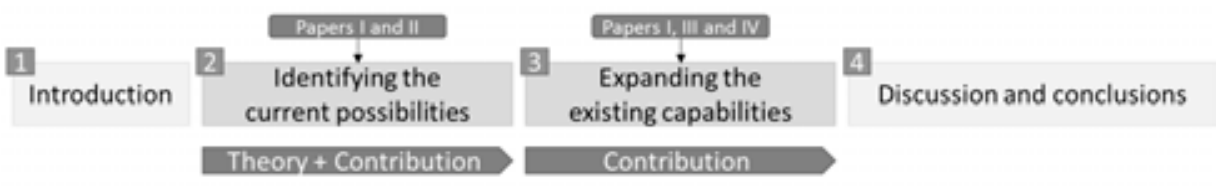

Figure 4. Overview and breakdown of the chapters.

A short summary of the four main chapters and their contents is presented below:

- Chapter 1: Presentation of the background as well as the research method, aim, and scope.

- Chapter 2: Focus on identifying the current MDO possibilities in UAV design (Overview of the state of the art, evaluation of the industrial adaptation and integration, presentation of the gaps and trends, exploration of the improvement directions).

- Chapter 3: Focus on the improvement of the existing MDO capabilities (Suggestion of a roadmap, development of an MDO framework, consideration of more efficient computing methods, presentation of optimization and validation results).

- Chapter 4: Discussions on the research contributions compared to previous works and presentation of the limitations as well as generalization of the proposed method. Conclusions with answers to the research questions and suggestions for future work. 


\section{2 \\ Identifying the Current Possibilities in MDO of UAVs}

The primary aim of this chapter is to provide the basic theoretical background that the reader needs in order to understand the technical terms and discussions which are presented later in this thesis. The chapter begins with the results of a brief literature review that was performed specifically for this thesis, and highlights the important subjects which have been omitted from the review papers but are nevertheless instrumental in understanding the particulars of the field. This "state of the art" section includes a small introduction to the principles of UAV design as well as engineering design optimization, and then it elaborates on two key MDO concepts which are namely the decomposition architectures and the efficient computing methods.

At a secondary level, this chapter aims to present a further and a more in-depth theoretical analysis of MDO in respect to the PDP of UAVs as well as the findings and the contributions of the review papers I and II. The purpose of the next sections is thus twofold, and it is to summarize the identified research trends in the form of further theory but also to provide an answer to RQ1 through the presentation of the research gaps that the literature revealed in the form of contributions.

In this light, the first topic is about the industrial adaptation of MDO as it was portrayed in paper II, and more specifically, about its potential to enhance the PDP, to promote better organizational integration, and to manage complex engineering systems. The next topic is about the gaps and trends in MDO of UAVs based on the findings of paper I, and in particular, about the possibilities in problem formulation, disciplinary modeling, analysis capabilities, and level of fidelity. Finally, the chapter concludes with a general assessment of the MDO field and sums up by presenting the directions for future improvement which were identified in both papers I and II.

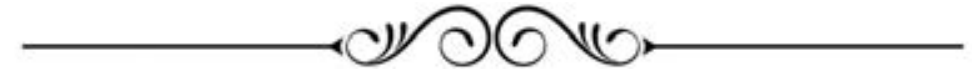




\subsection{State Of The Art}

This section is based on a literature review that was performed exclusively for this thesis, and presents the theoretical background which is required in order to follow this work. First, the target product is introduced by means of describing some key UAV concepts. Second, the basics of the method are explained through the presentation of the optimization concepts. Finally, the MDO topics that this thesis builds upon in the contributions are further elaborated.

\subsubsection{Design principles of UAVs}

The design of UAVs is a multidisciplinary process that begins with the definition of the general requirements and specifications which are typically a list of the most critical mission characteristics such as the payload, the endurance, the altitude, and the speed (Valavanis and Vachtsevanos, 2015). After this has been established, an initial sizing based on similar aircraft applications takes place in order to narrow down the potential airframe concepts, while the next steps are to investigate the aerodynamic efficiency of the chosen configuration, to establish a geometrical layout that has adequate volume for the systems, to calculate the generated structural responses based on the loading, and finally, to select a proper engine that meets with the minimum thrust requirements (See Figure 5). Similarly to general aviation aircraft, the ultimate goal herein is to be able to fly as efficiently as possible, and hence, one of the main design concerns is not only to develop flyable solutions but also to work towards better performance by reducing the aerodynamic drag and minimizing the engine fuel consumption (Austin, 2010).

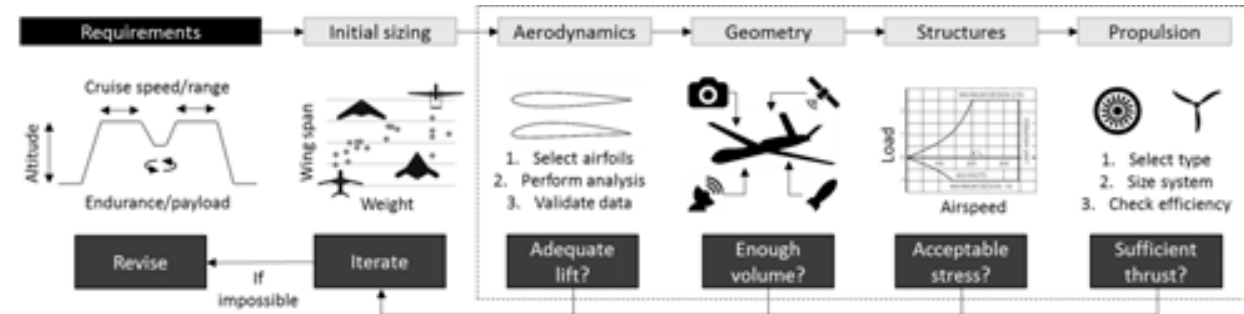

Figure 5. A simplified multidisciplinary development process showing the basic iterative loops in the design of UAVs.

From a systems architecture point of view, UAVs have the same components as manned aircraft with the main exception being that there is no need to have a cockpit or any kind of environmental control and life support systems (Austin, 2010). Although this is a significant weight saving, it is often compensated by the need for advanced guidance, navigation, and control systems which can be quite demanding depending on the size of the airframe and the desired level of autonomy (Valavanis and Vachtsevanos, 2015). In addition to these systems, a further weight penalty comes 
from the so-called "payload" which as a general rule defines the purpose of each UAV and it is usually comprised of various sensors, mission-specific items (e.g. commercial cargo, weapons), or a combination both. In their simplest form, the takeoff weight and the endurance of UAVs are respectively defined in Equations 1 and 2 where $C_{t}$ is the Specific Fuel Consumption (SFC) of the engine, and $L, D$ are the aerodynamic lift and drag forces. On the whole, Equations 1 and 2 illustrate the importance of a highperformance design, and point to the fact that a good aerodynamic as well as engine efficiency can reduce the fuel weight, increase the endurance, and subsequently allow for even more useful payload to be considered in the mission.

$$
\begin{gathered}
W_{\text {Takeoff }}=W_{\text {Airframe }}+W_{\text {Payload }}+W_{\text {Engine }}+W_{\text {Fuel }} \\
E=\frac{1}{C_{t}} \frac{L}{D} \ln \frac{W_{\text {Takeoff }}}{W_{\text {Takeoff }}-W_{\text {Fuel }}}
\end{gathered}
$$

\subsubsection{Engineering design optimization}

In a nutshell, engineering design optimization is a process that aims to improve the quality of the design by exploring how a representative set of design variables can affect a suitable set of objectives. Hence, the design variables can be viewed as the parameters that is possible to adjust in order to achieve the desired attributes, while accordingly, the objectives are mathematical expressions of the design characteristics that are expected to add "value" to the final product (Andersson, 2001). Overall, the system design and optimization process aims to support and speed up the development process, and to this end, it is essential to have a correct problem definition, adequate modeling as well as simulation capabilities, and finally a suitable optimization environment that can enable the evaluation of the various concepts (see Figure 6).

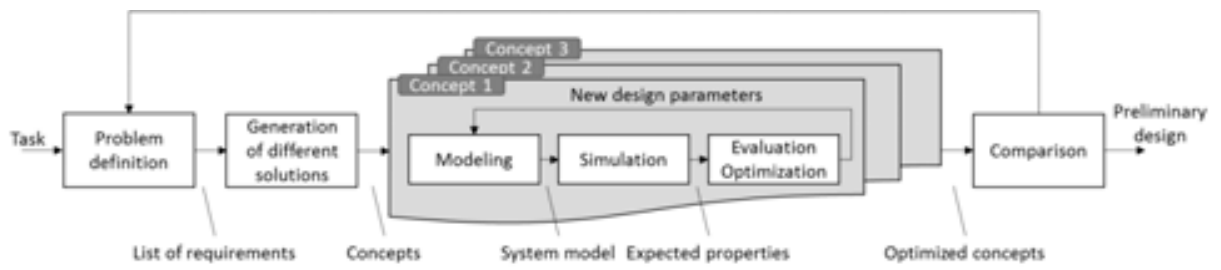

Figure 6. Graphical illustration of the system design and optimization process, adapted from Andersson (2001).

Apart from the above descriptive formulation, the typical design optimization problem can also be expressed in mathematical terms, and its basic form according to Sobieszczanski-Sobieski et al. (2015) that is also encountered in this thesis is presented in Equation 3. The considered problem takes into account a generic objective function which is denoted here as $f(x)$, two sets of inequality and equality constraints that are represented by $g_{j}(x)$ and $h_{j}(x)$, and lastly a set of design variables $x_{i}$ with $x^{u}$ and $x^{l}$ being the upper and lower limits respectively. 


$$
\begin{array}{cll}
\text { Minimize } & f(x) & \\
\text { Subject to } & g_{j}(x) \leq 0 & \text { for } j=1 \ldots m \\
& h_{j}(x)=0 & \text { for } j=1 \ldots p \\
& x^{u} \geq x_{i} \geq x^{l} & \text { for } i=1 \ldots n
\end{array}
$$

The optimization problem of Equation 3 can be solved in many ways depending on the particulars of each case-study, and to do so, it is first and foremost important to understand and interpret the requirements, to constraint the problem in a realistic way, and to keep the complexity at a level that corresponds to each development stage (Giesing and Barthelemy, 1998). To this end, some key concepts regarding problem formulation and optimization algorithms which are also used in the appended papers are elaborated below:

- Objective formulation: In a single-objective optimization (SOO) formulation there is only one characteristic that the algorithm seeks to optimize. This can be either a single attribute (e.g. the aircraft weight) or a combination of attributes that have been appropriately integrated into one aggregated objective function (e.g. aircraft weight, endurance, and cost). The latter can be formulated by using the weight-sum method which is a simple way of considering multiple objectives in the optimization problem by means of userdefined weightings (Andersson, 2001). An alternative to the above that gives more freedom to the decision-making team is a multi-objective optimization (MOO) formulation where two or more characteristics are optimized at the same time and eventually lead to a graph of equally optimal solutions which is known as the Pareto front (Savic, 2002).

- Optimization algorithms: A wide selection of both gradient and non-gradient algorithms have been applied to engineering design optimization (Sobieszczanski-Sobieski et al., 2015). A branch of the latter category that is also used exclusively in this work is the Genetic Algorithms (GA) which imitate the process of natural selection that occurs in nature. The design parameters are coded into genes which form chromosomes, and then those are evaluated so that the "fittest" can be identified and combined in order to produce an offspring (Goldberg, 1989). One of the strengths of GAs is their ability to locate the optimum even in cases that the objective function is not "well-behaved", whereas on the downside, they can be often computationally heavy due to the fact that they perform a more thorough exploration of the design space (Amadori, 2012).

In general, design optimization may be a straight-forward task when one discipline is considered, but at the same time, many challenges arise when it is applied on complex engineering products that are comprised of many sub-systems. Optimizing the 
sub-systems separately would most likely lead to sub-optimal or even unfeasible designs, and the main reason for this is that complex products have typically numerous synergies that need to be simultaneously taken into account (De Weck et al., 2007). Consequently, one possible solution is to move towards a more holistic representation of the system by including multiple disciplines (see Figure 7), and in this light, MDO is herein defined and used as a systematic approach to design space exploration that allows designers to map the interdisciplinary relations (Vandenbrande et al., 2006).

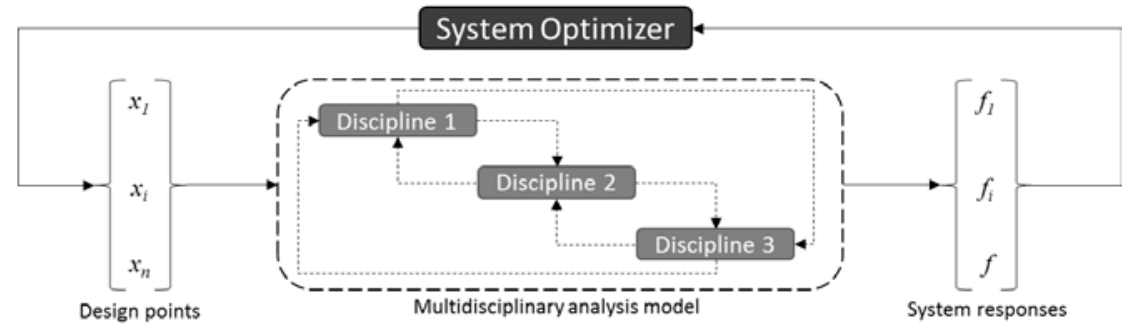

Figure 7. An example of an MDO framework with three disciplines, adapted from Vandenbrande et al. (2006).

\subsubsection{Decomposition of MDO problems}

The majority of MDO problems aim to capture the complex interactions of engineering systems, and therefore, a suitable decomposition "architecture" or "strategy" must be first established in order to be able to solve them. According to the definition of Martins and Lambe (2013), "architectures define how to organize the disciplinary analysis models, the approximation functions (if any), and the optimization software in concert with the problem formulation so that an optimal design can be achieved". In general, architectures are divided into monolithic (or single-level) and distributed (or multi-level) which in turn indicates that the formulation either considers only one main problem or multiple sub-problems that need to be coordinated at the same time. Overall, there are many different decomposition architectures that can be fitted to a variety of MDO problems, and to this end, some of the most common considerations are factors such as the complexity of the system interactions, the availability of specific algorithms, and the access to computer power.

In literature, the most fundamental monolithic MDO architecture is known as the "All-At-Once" (AAO) problem which includes all coupling variables, coupling variable copies, state variables, consistency constraints, and residuals of the governing equations in the problem statement (Martins and Lambe, 2013). Depending on which equality constraint groups are eliminated from the AAO problem, two other monolithic architectures can be subsequently derived, and those are namely the Individual Discipline Feasible (IDF) and the Multi-Disciplinary Feasible (MDF). Having established the above foundation, the next step is to derive the distributed architectures that are essentially an application of the IDF and MDF formulations on 
multiple sub-problems which are then typically controlled by a system-level problem (see Figure 8).

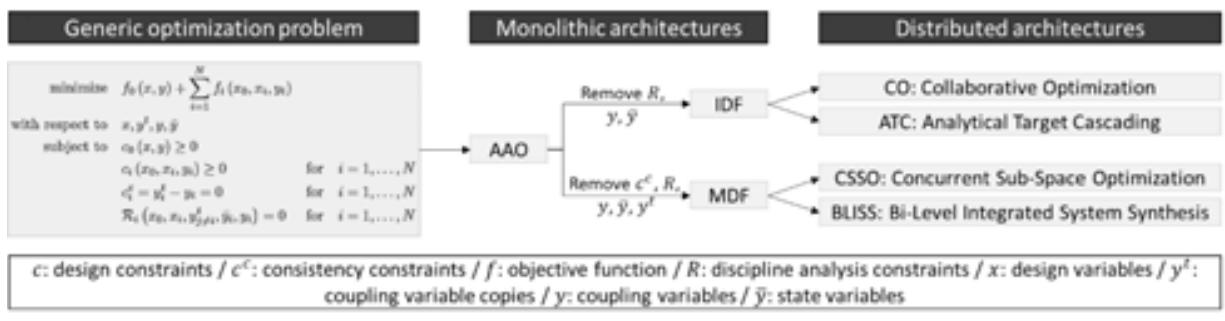

Figure 8. The derivation of the fundamental MDO decomposition architectures, adapted from Martins and Lambe (2013).

As far as monolithic architectures are concerned, the MDF is the simplest to implement, while at the same time, it also ensures that there is always system consistency even if the optimization process is terminated early (Balesdent et al., 2011). In MDF, all the sub-systems are coupled together in an analysis module that receives the design variables $x$, then iterates with the discipline outputs $y_{i}$ and the state variables $z_{i}$ until convergence has been reached, and finally calculates the objective function $f$ as well as the equality $h$ and inequality $g$ constraints (see Figure 9 left). The convergence loops of MDF are based on fixed-point iterations which require multiple disciplinary analyses for each one of the global algorithm evaluations, and for that reason, a significant amount of computational time is often spent in this process. Thus, the MDF is more suitable for smaller problems where fast analysis times are expected, while to this date, in aircraft MDO it has been typically restricted to the decoupling of a small number of disciplines like for example propulsion and mission performance (Allison et al., 2012) or structures and aerodynamics (Brezillon et al., 2012).
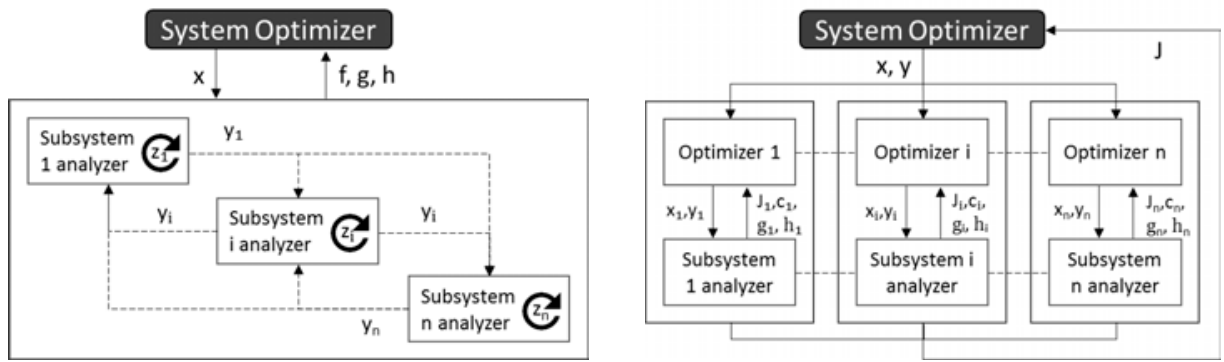

Figure 9. The MDF (left) and CO (right) decomposition architectures, adapted from Balesdent et al. (2011).

A distributed architecture that is based on the IDF formulation and has been frequently applied in aircraft MDO for achieving mission-based (Perez et al., 2006) or discipline-based (Iwaniuk et al., 2016) decomposition is Collaborative Optimization $(\mathrm{CO})$. $\mathrm{CO}$ divides the problem in many different parts which are then controlled by a global optimizer, and in this respect, it has the main advantage of enabling a better 
problem decoupling and allowing disciplines to be analyzed in parallel (Balesdent et al., 2011). In CO, all the sub-systems receive the design as well as coupling variables $x, y$ and then modify their local copies subject to the local constraints $h_{i}, g_{i}$ as well as the coupling functions $c_{i}, J_{i}$. Once all the local optimizations are finished, the system optimizer evaluates the objective function but also the consistency residuals $J$ in order to determine the improvement of the objectives but also the achieved decoupling level (see Figure 9 right). Due to its complex nature, CO typically requires a significant overhead development time, while a further computational weakness is that consistency and feasible solutions are not always guaranteed if the user decides to abruptly stop the process before the optimizer has completely converged.

\subsubsection{Efficient computing methods}

One of the most important characteristics that every MDO framework should have is the ability to provide quick answers so that it can increase the design knowledge at an even earlier stage in the development process. In this light, one frequently implemented strategy is to use surrogate models or metamodels in order to replace the computationally expensive disciplinary analyses (Giesing and Barthelemy, 1998). Generally, metamodels are mathematical functions and they are created by first identifying the response of the original model over a predefined design space, and then applying an approximation algorithm in order to be able to capture its behavior (Viana et al., 2014). According to Myers et al. (2009), there are many approximation algorithms for creating metamodels, and some of the most notable ones that are also used in this work include Anisotropic Kriging (AKR) and Neural Networks (NN) which are further elaborated below.

- AKR belongs to the field of geostatistics, and calculates the value of the desired point based on its distance to a set of other known points and the overall trends of the function in the given design space.

- NN are inspired by how the human brain processes incoming information, and they are based on a grid of several hidden layers which aim to relate the input to the output by using simple transfer functions.

Nevertheless, metamodels can also have many disadvantages, and in particular, the most common issue is that their predictions can have a significant deviation from those of the real models. Depending on the type and scope of the application, even a large error can be sometimes acceptable, while in general, it can be said that the most influencing factors which can affect the final accuracy are the number of the input variables, the amount of noise in the function, and the quality/size of the training sample (Persson, 2015). To this end, there are many authors who have investigated various methods to increase the performance of the metamodels, and the most notable examples are to recalibrate the metamodels after each iteration (Lefebvre et al., 2012), to limit their predictions only at a narrow area of the design space (Choi et al., 2008), 
and lastly, to decompose the problem into smaller parts and then use multiple metamodels (Piperni et al., 2013).

\subsection{Industrial Adaptation}

This section covers the issues which are pertain to the current relation of MDO to the manufacturing industry, and more specifically, to the particulars of its cultural and organizational integration within the PDP. The discussions herein are solely based on the findings of the review that is presented in paper II, and the three presented topics have been chosen in order to illustrate the non-technical potentials of using MDO within a generic PDP.

\subsubsection{Enhancing the development process}

A well-defined process is an essential element of product development, and it has been shown that it can lead to increased coordination, better planning, and continuous design quality improvement (Ulrich and Eppinger, 2012). Like many other complex engineering products, the PDP of UAVs begins with planning and it goes through many development phases before the design is ready to be tested and put in production (see Figure 10). In the design stages of this generic PDP several different airframe configurations are initially explored (conceptual design), and once a suitable solution has been identified, then each component of the system is gradually refined (preliminary design) until a complete engineering drawing can be delivered (detailed design). Overall, the challenge in conceptual design is to be able to quickly explore several airframe configurations in order to find a layout that generally meets with the requirements, while in the preliminary and detailed design it is important to include more in-depth disciplinary analyses so that the operation and key interactions of each sub-system can be accurately captured.

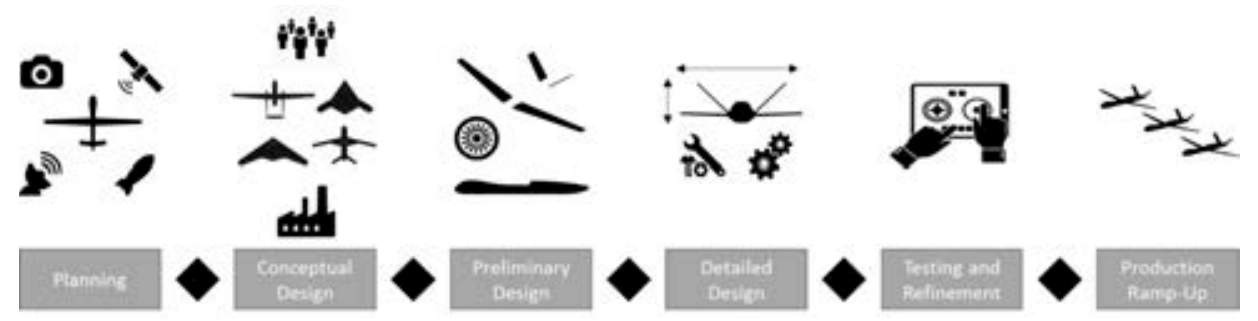

Figure 10. The typical design stages in a generic development process for UAVs, adapted from Ulrich and Eppinger (2012).

As already stated before, MDO has the potential to bring more knowledge into the process, and hence, it can be seen that it has been successfully applied on all stages of the PDP as well as between different departments of the organization like marketing, engineering, and manufacturing. Conceptual design is by nature a very promising field 
of application for MDO, and it has been shown in many instances (Amadori et al., 2006; Jeon et al., 2007) that it can be a quick tool for exploring the underlying tradeoffs between competing objectives in UAV design. Accordingly, MDO can have many uses in preliminary design where it can lead to a more holistic view of the total system behavior by taking into account the effects of the on-board systems like the propulsion (Allison et al., 2012) or the flight controls (Perez et al., 2006). Lastly, there are traditionally further uses of MDO in the detailed design stage, and more specifically, it can be an effective method to either refine the design with greater accuracy or provide more data earlier in the process through the use of increased problem sizes and high-fidelity simulations (Choi et al., 2010).

\subsubsection{Achieving organizational integration}

One of the most critical issues towards achieving products of higher quality is to be able to bring the different departments of the organization closer and in turn use this integration in order to enhance the development activities with more efficiency and more professionalism (Andreasen and Hein, 1987). The main problem here is that modern companies grow larger with time, and hence, this causes a segregation of the organizational departments which in turn reduces the communication and affects the final product quality (see Figure 11). To this date, changes in the organizational structure as well as in the topology of the facilities are the most common strategies to tackle this problem (Griffin and Hauser, 1996), however, this is not always the solution for large UAV companies where their size can be often a major hinder towards integration.

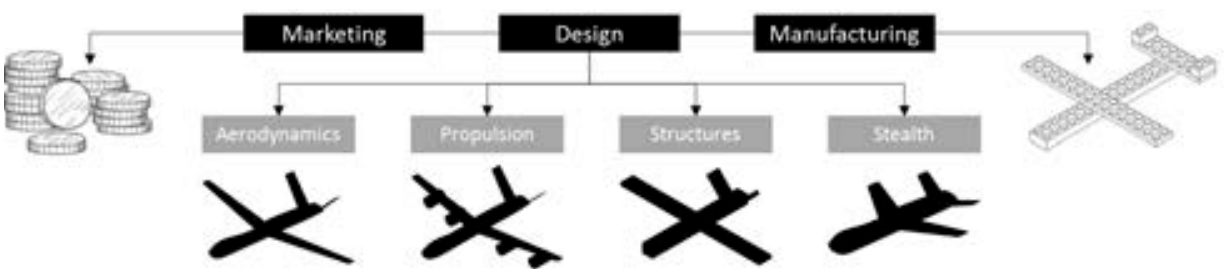

Figure 11. An example of the different department desires within the PDP of UAVs, adapted from paper II.

In this respect, MDO can be a decisive factor since it has been shown to bring people from all departments and hierarchies closer in order to work towards the development of a framework that can be used in the optimization of the product. In an MDO scenario, the disciplinary models should be able to exchange information seamlessly, and for that reason, there is an additional motivation for people to come together and interact much more often compared to the traditional PDP structure (Safavi et al., 2012; Safavi et al., 2015). This new state allows all departments and teams to see clearly the main design objectives, and as a direct consequence, it generates a state of increased awareness that is argued to reduce the costly and timeconsuming iterations within the groups (Simpson and Martins, 2011). 


\subsubsection{Managing complex systems}

Systems engineering is an iterative process of top-down synthesis, development, and operation that aims to solve the given problem in an interdisciplinary and sociotechnical approach while considering the entire life-cycle of the product (Haskins et al., 2006). In the development of UAVs, the main challenge for designers is to balance multiple factors like for example the cost, the scheduling of the process, the quality of the product, the future changes, and the financial risk (Crawley et al., 2004). Hence, there is clearly a need to model more system aspects, but also to consider more lifecycle inputs and outputs so that further knowledge regarding the functional behavior and the potentially undesirable effects can be taken into account as early as possible in the design decisions of the PDP (see Figure 12).
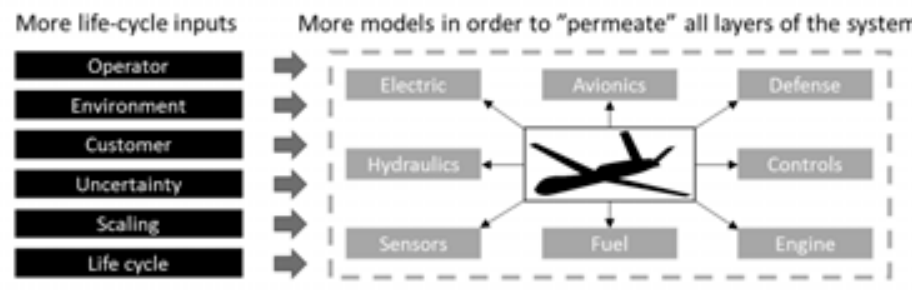

More life-cycle outputs

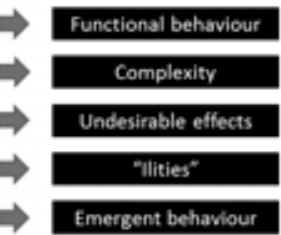

Figure 12. An ideal MDO approach for the design of UAVs by considering all layers of the system as well as life-cycle aspects, adapted from paper II.

Given the above definitions, it can be argued that MDO can be a valuable tool for acquiring a holistic view of the UAV systems since it can permeate all layers of the design through the use of concurrent airframe and sub-system evaluations in a suitable computational environment (Krus and Andersson, 2003). Moreover, MDO can ensure that additional information on properties such as robustness, adaptability, flexibility, scalability, and safety are also delivered by using suitable inputs to the problem like for example probabilistic and uncertainty constraints (Gavel et al., 2008). Finally, considering logistics and marketing is also a further possibility, and it can be seen that designing families of aircraft is one example which shows how modularity and scalability can be effectively included in the MDO framework (Willcox and Wakayama, 2003).

\subsection{Gaps and Trends}

This section presents the current research gaps and trends which were identified by the survey of paper I. The literature sample for this review consisted of $67 \mathrm{MDO}$ case studies in aerial vehicle design which were carefully selected after following a stringent methodology. The most important results of this review are summarized here in four sub-sections, while the interested reader can find the complete and detailed work in the appended papers. 


\subsubsection{Problem formulation}

In the optimization of aerial vehicles, the most common objectives are related to the mission, weight, and aerodynamic performance of the design, and the reason is that those characteristics can be a good overview of the general product value (see Figure 13). According to the review that is presented in paper $I$, the most frequent formulation is the SOO, whereas multiple objectives can also be taken into account by using the weighted-sum method or a MOO which is a more flexible alternative for design space exploration (Hurwitz et al., 2012). Moreover, the most preferred metrics are typically design parameters which are associated with the weight of the aircraft (e.g. MTOW) due to the fact that those are often related to the overall aircraft performance as well as the operating and acquisition costs (Ghoman et al., 2012).
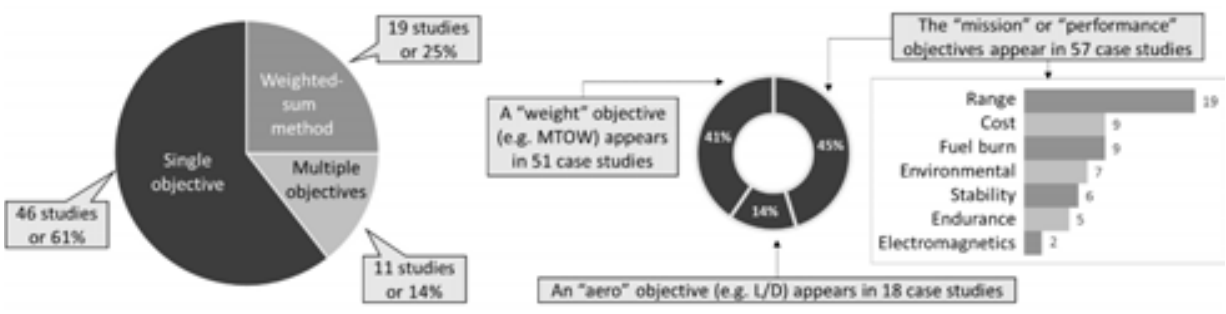

Figure 13. The types of formulations and objectives in MDO of UAVs, adapted from paper I.

\subsubsection{Disciplinary modeling}

In general, the number and complexity of the models are aligned with the requirements of each design application, and in this respect, the standard practice is to consider a set of common aeronautical disciplines that can simulate the basic aircraft performance but also several alternatives that can capture the case-specific physics (see Figure 14).

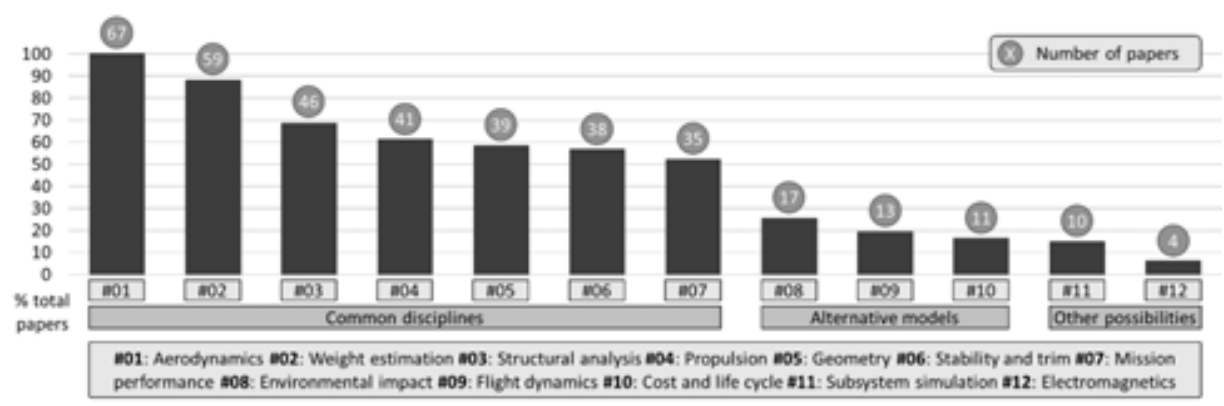

Figure 14. The common disciplines and the possible alternatives, adapted from paper I.

The common aeronautical disciplines are typically expressed in conceptual design with low fidelity tools or empirical equations, while in the more detailed design application it is usually necessary to implement higher-fidelity tools in order to enhance the accuracy of the calculations. Overall, the disciplines can be used either in a "stand- 
alone" or "support" mode depending on their function, and a typical example of the latter in MDO are the geometry models which are often employed in order to provide more detailed information to the other analysis elements of the framework (Laban and Herrmann, 2007).

According to paper I, there has been extensive research on the common disciplines and their integration in the MDO framework for the design of aerial vehicles, but at the same time, there is a research gap in respect to the alternative models which in turn calls for more disciplines to be gradually included as suggested by Agte et al. (2009). Thus, for UAVs it is generally important to include noise propagation models that show the sound impact of the design towards the ground observers (Choi et al., 2008), flight mechanics models that simulate the interactions between the control system and the behavior of the aircraft (Haghighat et al., 2012), and cost models that can estimate the economic implications and life-cycle evolution of the product (Sadraey, 2008). Finally, further additions which are typically neglected but are especially critical for surveillance UAVs are the simulation of the on-board sub-systems which can provide more data on the system interactions (Piperni et al., 2013), and the modeling of electromagnetics which can capture aspects such as the communications (Neidhoefer et al., 2009) and the stealth performance (Allison et al., 2012).

\subsubsection{Analysis capabilities}

The term analysis capabilities aims to describe complex computational functions that are able to provide additional information on the design by using the existing framework models (see Figure 15). One indicative example of this is the calculation of the aeroelastic equilibrium which to this date has received, and still receives, a lot of attention since it is a crucial aspect in the design of aircraft with flexible wings (Cavagna et al., 2011).

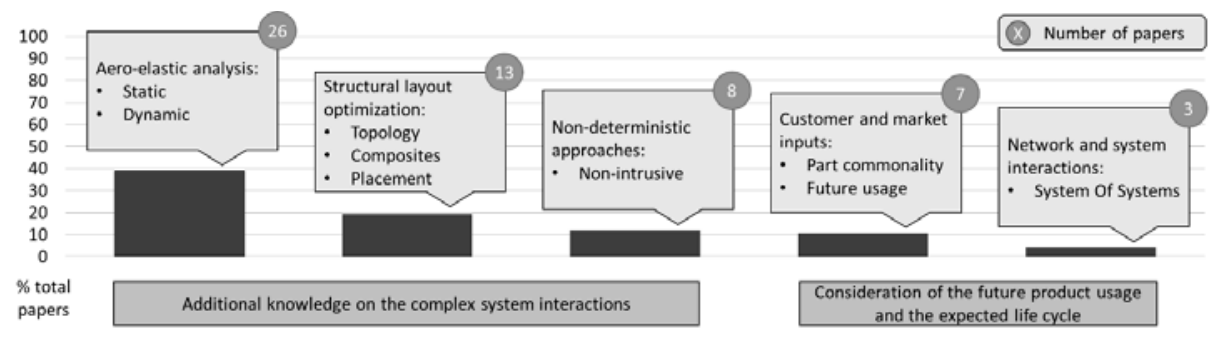

Figure 15. The typical analysis capabilities in MDO of UAVs, adapted from paper I.

Accordingly, in the design of aerial vehicles supplementary fidelity can be brought into the conceptual design by implementing a local high-fidelity optimization of the structural layout (Gazaix et al., 2011), while additional knowledge regarding the robustness and the unwanted system effects can be achieved through the consideration of uncertainty inputs (Yao et al., 2011). Lastly, in the fast-paced development market of UAVs it is important to be able to have a holistic view of the future product usage 
early on, and in this light, two essential, but yet overlooked, analysis capabilities are to be able to predict the customer and market inputs as well as the network and system interactions.

\subsubsection{Level of fidelity}

The level of fidelity that the disciplinary models should deliver is primarily determined by the development stage which the MDO process aims to enhance, and therefore, it is always crucial to take into account the degree of design maturity that needs to be achieved (Piperni et al., 2013). According to the review of paper I, the majority of authors abstain from specifying the development stage that they are working on, while at the same time, there is general tendency where the choice of tools is based on availability rather than suitability. Overall, it is stressed that the MDO tools should be able to capture the correct physics of the problem (Reuter et al., 2016), but also to be as computationally efficient as possible in order to enable even faster design evaluations (Henderson et al., 2012). To this end, it can be seen that a prevalent trend is to build modular frameworks that can adapt to different fidelity requirements, whereas it can be argued that the main gap herein is the lack of a complete list regarding the available software solutions (see Figure 16).

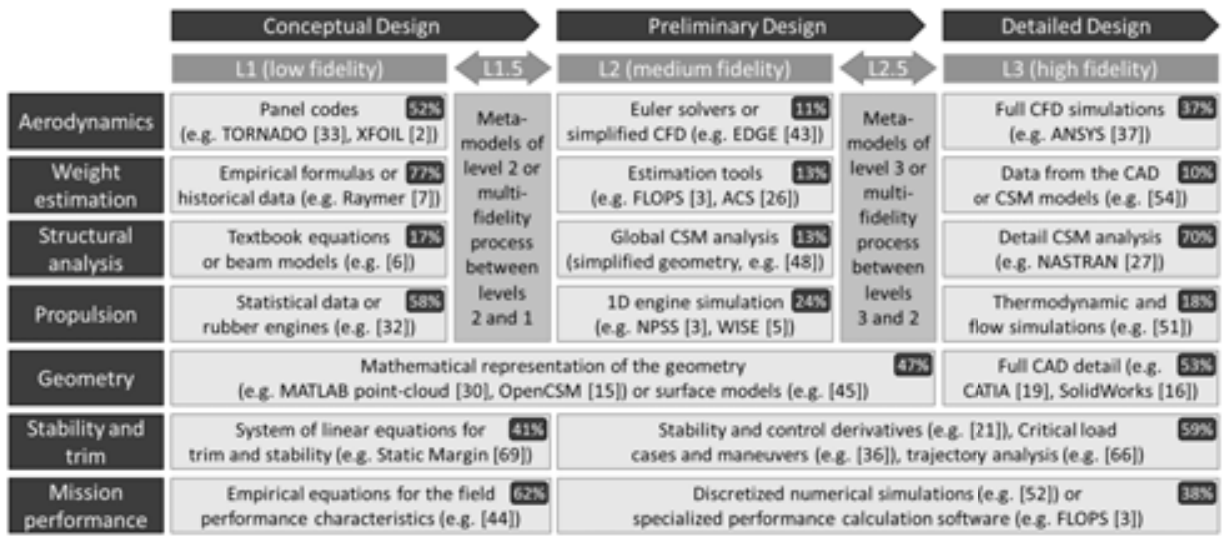

Figure 16. The tools that are commonly used in MDO of UAVs, adapted from paper I.

In total, low-fidelity tools are the most frequently used solution in MDO for conceptual design of UAVs (see Figure 16), and the main reason for this is that they can deliver a sufficient level of fidelity at very fast computational times (Iemma and Diez, 2006). Nevertheless, low fidelity has many disadvantages, and more specifically, it can be seen that it is often impossible for elementary solutions like empirical equations to capture the complexity of detailed design or unconventional configurations (Allison et al., 2012). Thus, in such cases it is imperative to employ higher-fidelity models or their metamodels which can be an efficient way of increasing the confidence on the design at a reasonable loss of accuracy. Finally, considerable research has also been conducted on multi-fidelity schemes, and in particular, it has been shown that a 
very efficient alternative is to couple a low fidelity process with a high-fidelity one. In this way, it is possible to enable a first and fast exploration of the design space, and then perform the more detailed and thus time-consuming search on a much narrower area where this is truly needed (Zill et al., 2013).

\subsection{Improvement Directions}

In this section, the identified directions for improving the MDO field are presented based on the findings and discussions of papers I and II. The focus is initially on the general shortcomings of MDO and the challenges towards its integration within the PDP. At a secondary level, the purely technical limitations of MDO are presented and the possibilities for expanding the traditional UAV design frameworks are finally elaborated.

\subsubsection{General research assessment}

A general research objective in MDO that is shared by the community since the first reviews of Sobieszczanski-Sobieski and Haftka (1997) is to enable calculations of even higher fidelity while at the same time reducing the computational demands. Although significant steps have been achieved in this direction, there are still several research gaps in terms of expanding $\mathrm{MDO}$, and one of the most critical shortcomings according to paper $\mathrm{I}$ is still the breadth and depth of the disciplinary modeling as it has also been previously reported by Agte et al. (2009). In addition to this, the review of paper II identified that there are also several hinders towards implementing MDO in the PDP, and as already stated in the work of Simpson and Martins (2011), there is still a shortage of technical publications, absence of MDO in higher education, uncoordinated research, problems with industrial adaptation, and barriers in the transmission of knowledge due to corporate secrecy (see Figure 17).

According to the literature review of paper I, two typical examples of modeling limitations are the front and back end of the PDP, and more specifically, it can be seen that there is need to enhance the traditional MDO practices with more details regarding the manufacturing process, the product maintenance, the operating environment, the market evolution, and the intangible entities like the aesthetics of the design. To this date, there have been several efforts to expand the traditional MDO frameworks with new disciplinary models like noise impact, flight mechanics, and cost estimation, but those have been studied in isolation rather than in a concurrent manner that will enable a holistic assessment of the design. Accordingly, there is a need to simultaneously include even more analysis capabilities in order to expand the applicability of the framework, and in this respect, it can be seen that features such as aeroelasticity, structural optimization, and uncertainty inputs can be instrumental in understanding the total behavior of the system. 


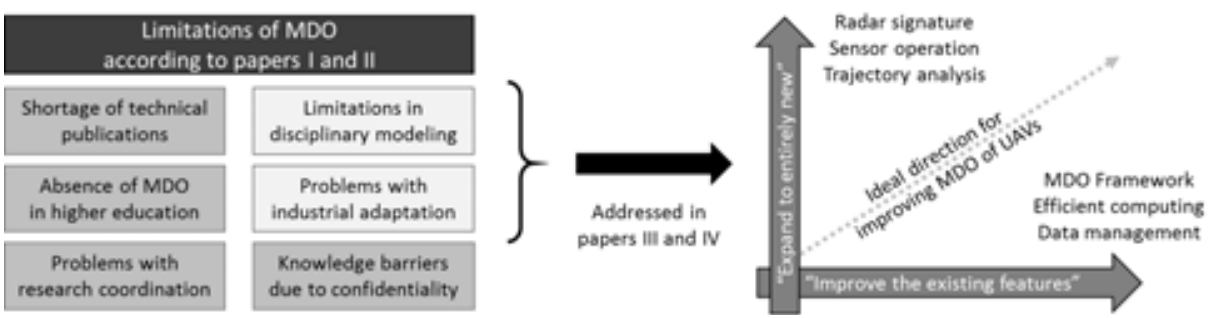

Figure 17. The research gaps and improvement directions, adapted from Agte et al. (2009).

\subsubsection{Possibilities in UAV design}

As far as surveillance UAV applications are concerned, two key design aspects that can drive the design are to be able to estimate how well the sensor system is performing and how observable the aircraft is to the ground radars. Nevertheless, according to the literature, the operation of the on-board aircraft systems is seldom included in the optimization frameworks, while the radar signature has only been assessed in oversimplified scenarios. In addition to this, as a means to express the customer demands and the future operating environment, it is important to be able to take into account all possible aircraft usages, and therefore, a function that can add further knowledge into the design is to include a trajectory analysis of the anticipated surveillance scenario.

In this light, the contribution of this research focuses primarily on expanding the traditional optimization framework with more modeling features as well as more analysis capabilities in an effort to be able to apply MDO on UAV case studies that consider surveillance requirements. At a secondary level, the emphasis is on developing methods to support the integration of MDO within the industry, and to this end, it is investigated how the framework performance can be further improved and how the results can be used in a meaningful way by the decision-making team. Overall, the improvement possibilities herein have been divided into two orthogonal directions as suggested by Agte et al. (2009) with the horizontal being about improving the existing practices and the vertical about extending the general possibilities (see Figure 17):

- Horizontal expansion: development of an MDO framework for a generic UAV design by using an alternative set of tools for the common aeronautical disciplines; exploration of known efficient computing solutions for MDO like for example asymmetric architectures and multiple metamodels; assessment of data management as well as visualization tools.

- Vertical expansion: consideration of aspects that have been overlooked but at the same time can be vital in MDO of surveillance UAVs; development of electromagnetic models like radar signature and system operation models like sensor performance; expansion the framework with inputs such as customer demands and network interactions through the trajectory. 


\section{Expanding the Existing Capabilities in MDO of UAVs}

Given the findings of the literature review which were presented in the previous chapter and in paper I, the next part of this research is to implement that knowledge in order to synthesize a roadmap that can systematize the process of applying MDO on UAVs. Having established this foundation, the next step is to apply the suggested methods on an optimization case study, and more specifically, to validate it through the development of an MDO framework that can be used in the conceptual design stage of UAVs.

On the whole, the main objective of the proposed framework is to assess the efficiency of the current practices in MDO, while at a secondary level the intention is to create a solid basis that will allow an expansion of the existing capabilities. In particular, surveillance and survivability over inhospitable territory are two of the most desired UAV attributes (Volpe, 2013), however, it can be argued that those design aspects have been seldom included in MDO frameworks. Consequently, it is essential to develop further models and capabilities that can provide metrics on those requirements, whereas it is equally important to enable methods that can align the performance of the tools with the corresponding design stage.

Overall, the proposed expansions are based on the research work that is presented in papers III as well as IV, and the general aim herein is to provide answers to RQ2 as well as RQ3 by assessing a number of potential improvement strategies for surveillance UAVs. The effect of the proposed additions is measured through the use of quantitative results from two exploratory case studies, while comparisons to the existing literature are presented throughout the text in order to qualitatively assess the discussed concepts. 


\subsection{Implementation Roadmap}

This section presents a roadmap for mapping the process of applying MDO in the development of UAVs. The roadmap structure is the first topic of this chapter in order to facilitate the understanding of the framework, the expansions, and the results that are discussed in the following sections. In this section, the work is based on the literature review of paper I and it is presented as a personal contribution regarding the methods of improving the PDP (answer to RQ3).

\subsubsection{Overview of the structure}

Given the state of the art that was presented above and that is further elaborated in paper I, the next step of this research is to contribute by introducing a roadmap regarding the implementation of MDO in UAV design (see Figure 18).

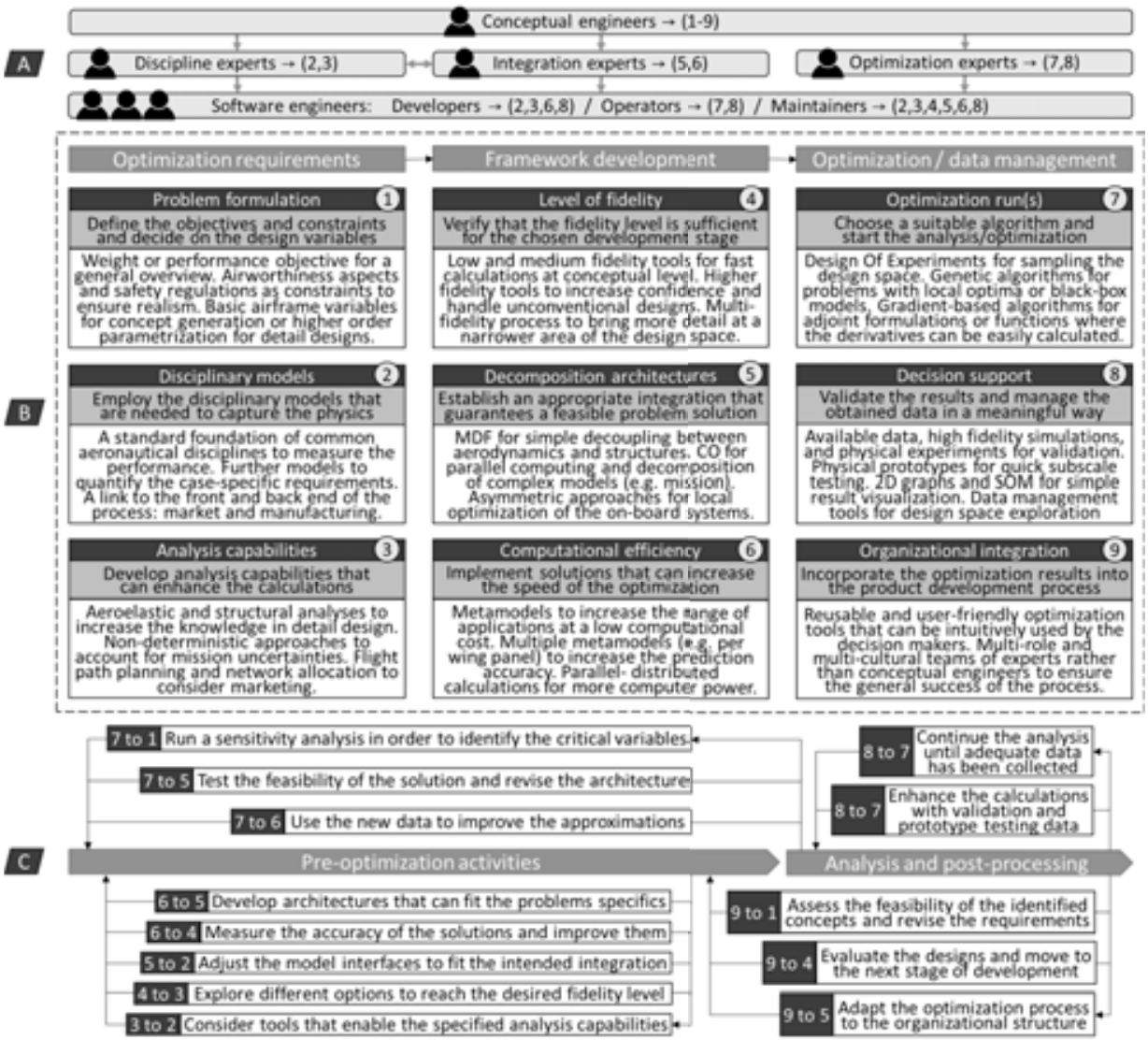

Figure 18. The proposed roadmap for applying MDO on UAVs, adapted from paper I.

The roadmap is comprised of three different and distinct blocks that are denoted as $\mathrm{A}, \mathrm{B}$, and $\mathrm{C}$ which aim to respectively describe the organizational needs, the 
fundamental elements, and the process iterations. Overall, the roadmap has been primarily based on the current developments, however, the work herein has also been enriched with the potentially critical elements that were identified during the investigation of the research gaps and trends. The main applicability of the roadmap is on UAVs, but it is proposed as a generic framework so that products with different design purposes like for example surveillance, rescue, agriculture, and military can be easily considered. The aim of the roadmap is to create a guideline that will promote the use of MDO in both academia and industry, and thus, it should be viewed as a first approach on how the practices of this active and dynamically changing research field can be modeled in a flexible but yet illustrative way.

\subsubsection{Description of the blocks}

In Figure 18, block (A) presents the people that should be involved in the MDO process, and the proposed solution is a combination of three different layers of experts which is based on the work of both Safavi et al. (2015) as well as Berends et al. (2006). Compared to the existing studies where the focus is either on engineering or software responsibilities, this new approach enables the consideration of scientific knowledge from three different groups of people which are namely the conceptual engineers, the domain experts, and the software developers. In this setting, the conceptual engineers are responsible for guiding the overall project and communicating the central objectives, while the technical tasks of model development, integration, optimization, data management, and system maintenance are delegated to the domain experts and software developers respectively.

Block (B) is comprised of 9 elements that correspond to the foundation of every MDO process. Each element has a specific topic and it starts by giving a clear instruction on what has to be done which is then followed by recommendations as well as possible alternatives that have been identified by the review of paper I. Here, the aim is to allow the user to efficiently navigate through a number of distinct steps that are usually taken into account in MDO of UAVs, and in turn to standardize the process by ensuring that all the critical elements have been considered. In this version of the roadmap, steps 1 to 3 are about setting up the problem based on a list of requirements, steps 4 to 6 are about integrating the models and analysis capabilities based on a suitable strategy, and lastly, steps 7 to 9 are about the process of optimization and management of the results.

Finally, block (C) summarizes the potential activities that can take place during optimization, and it is a personal research work regarding the iterative steps that should be ideally considered in order to improve the process based on the collective and critical assessment of the literature. The iterative steps of the proposed roadmap show the possible feedbacks that exist according to paper I, and the aim is to document the major activities of the process but also to provide the user with sufficient enhancement alternatives throughout the development of an MDO framework. The 
logic system that is used here is intentionally simple in order to allow for more clarity during the implementation of the suggested guidelines, while the notation has been formulated as a "message" which indicates that at the end of step X the user should go back to step Y and make the necessary changes.

\subsection{Framework Development}

This section focuses on the elements that comprised the MDO framework which was used in the two exploratory case studies. The aim here is to elaborate on the technical additions that were considered the most critical towards the improvement of the UAV optimization process (answer to RQ2). In this section, the work is based on the contributions of papers III as well as IV, and it includes the development of further disciplinary models and novel analysis capabilities.

\subsubsection{Basic and case-specific models}

The first consideration of the proposed MDO framework is to include disciplinary models that will enable the simulation of the performance in the context of a complex mission rather than just isolated flight conditions. Mission analysis is typically a function that depends on the output of many disciplines, and it can be a very representative metric of the airworthiness requirements and the field performance of the chosen design configuration. In conceptual design, a commonly accepted methodology is to use low-fidelity tools that allow for fast computational times (Amadori, 2012), and for that reason, complex CFD as well as CSM simulations were not considered relevant for this application. In total, the proposed framework includes models for aerodynamics, propulsion, stability, and weight estimation, while a CAD geometry model was also added as a support in order to enhance the calculations of the other disciplines.
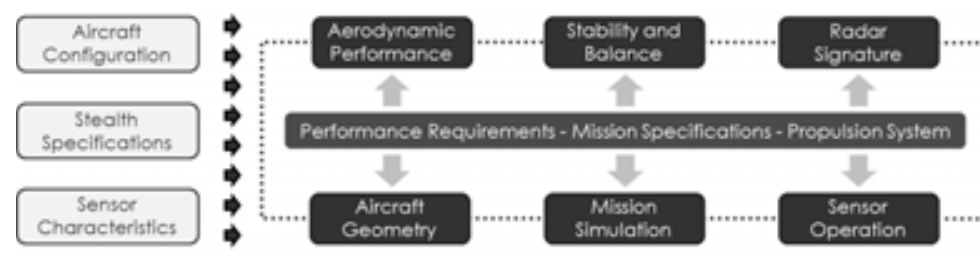

Figure 19. Overview of the UAV optimization framework, adapted from paper III.

The second objective of this work is to expand the above basic framework by including models that can capture the requirements of surveillance and survivability (see Figure 19). Although, there are many interpretations of the above concepts, one simple representation that is also used in this context is to express them by means of a model for simulating the effectiveness of the sensor system and a model for measuring the stealth characteristics. As expected, the sensor system is bound to have conflicting 
interactions with the propulsion unit due to cooling and power requirements, whereas a stealthy design can often have a negative effect on the aerodynamic efficiency but also the total structural weight.

For this conceptual design application, the sensor performance model was developed in MATLAB by implementing a set of appropriate electromagnetic equations that are readily available in literature (Balanis, 2002). Further possibilities include the use of finite element models as shown in (Neidhoefer et al., 2009), but this level of fidelity is disproportional to the general capabilities of the framework and therefore it can be avoided so as to maintain a reasonable level of optimization performance. Overall, in both papers III and IV a generic flat antenna type that can be integrated in the skin of the aircraft without any protruding objects was considered in order to define and in turn calculate the properties of the emitter and the receiver. The main objective here is to be able to direct the radiation of the sensor towards the angular sector where the target is located, and an indicative metric of this is the directive gain $D_{g}$. The directive gain is thus a measure of energy intensity, and in this respect, it can be further manipulated by using the radar-range equation so that it can provide other meaningful metrics such as the effective detection range or the target detection probability (see Figure 20). In the simplest form of this problem that is presented in paper III, the position of the target is assumed to be fixed which requires only one analysis per iteration, but if spatial information is available like in the more advanced application of paper IV, then multiple and thus time-consuming computations are typically necessary to complete the calculations.

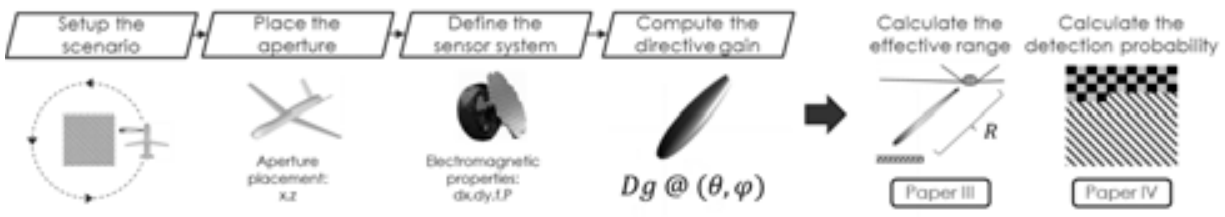

Figure 20. The functions of the sensor performance model, adapted from paper III.

The stealth performance is usually expressed by the Radar Cross Section (RCS) of the aircraft which is measured in square meters and corresponds to the size of the aircraft area that the ground radars will eventually "detect" (Raymer, 2012). The RCS cannot be measured adequately with empirical equations because it depends highly on a detailed representation of the design geometry, and for that reason, complex optical codes that require a significant amount of computational time are often necessary in order to accurately predict it (Allison et al., 2012). Hence, for this case study the Physical Optics (PO) tool GRECO (Rius Casals et al., 1993) is used as a basis for all the simulations to better capture the physics of the problem, and then it is integrated into the conceptual design framework by using the undeniably faster, but yet less accurate, alternative of metamodels. To further decrease the computational time, a common simplification is to reduce the "threat" angular sectors to either one (Mäkinen et al., 1999) or two (Tianyuan and Xiongqing, 2009), while a technique that has also 
shown promising results is to simulate a small number of points and then make predictions based on interpolation (Lee et al., 2008). For reasons of simplicity and speed, the approach of analyzing one sector is followed in the first and elementary case study that is presented in paper III, whereas the more intricate strategy of multiple simulations is followed in the second and more advanced case study of paper IV (see Figure 21).

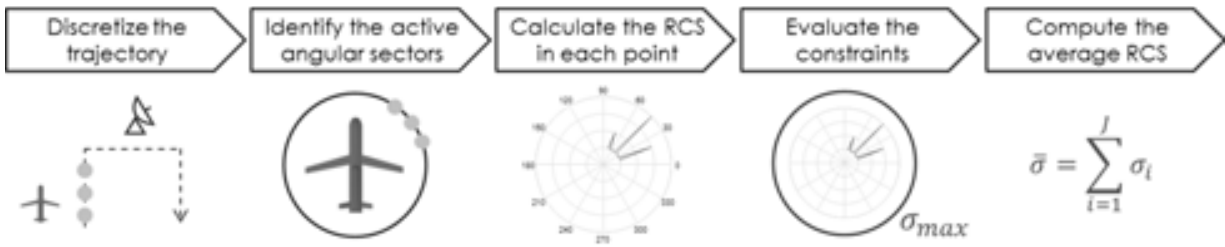

Figure 21. The calculation of the RCS by using multiple points, adapted from paper IV.

\subsubsection{Advanced analysis functions}

The analysis capabilities that have been taken into account in this work are about increasing the knowledge of the product during conceptual design, and this is addressed here through the consideration of more information regarding its future operation. The main challenge and motivation is that the response of the sensor and stealth models depends strongly on the view angles $(\vartheta, \varphi)$, and for that reason, it is often necessary to introduce a specific three-dimensional scenario in order to be able to simulate them throughout the entire mission. As already described above, this is commonly done by indicating one or more critical directions of interest, however, this is clearly a big simplification because in reality the ground radars and surveillance targets can appear in different angular positions. Consequently, the surveillance performance and survivability of the aircraft will ultimately depend on the view angles that are "activated" within each scenario, and as a result, there is a direct need to enhance the predictions of the models by including an analysis of the flight trajectory into the optimization problem.

To this date, the analysis of the trajectory in MDO has been used in only a few case studies in order to either identify the mission performance (Yan et al., 2012) or the radar constraints (Norsell, 2005), but the main limitation is that it has been restricted to two-dimensions and low fidelity mathematical models. The common approach that is also followed in this work is to discretize the trajectory into several segments, and then perform an evaluation of the design at each one of them. By enabling the MDO framework to consider this information, it becomes possible to have a holistic assessment of the trajectory-depended models, while at the same time, the decision-making team can also have access to further knowledge regarding the performance of the product under specific conditions. In the case study that is presented in paper III the analysis takes place at only a small number of predefined critical directions (see Figure 22 left), whereas in paper IV, a more advanced strategy 
that can simulate the surveillance scenario is proposed and evaluated against four different search trajectories (see Figure 22 right).
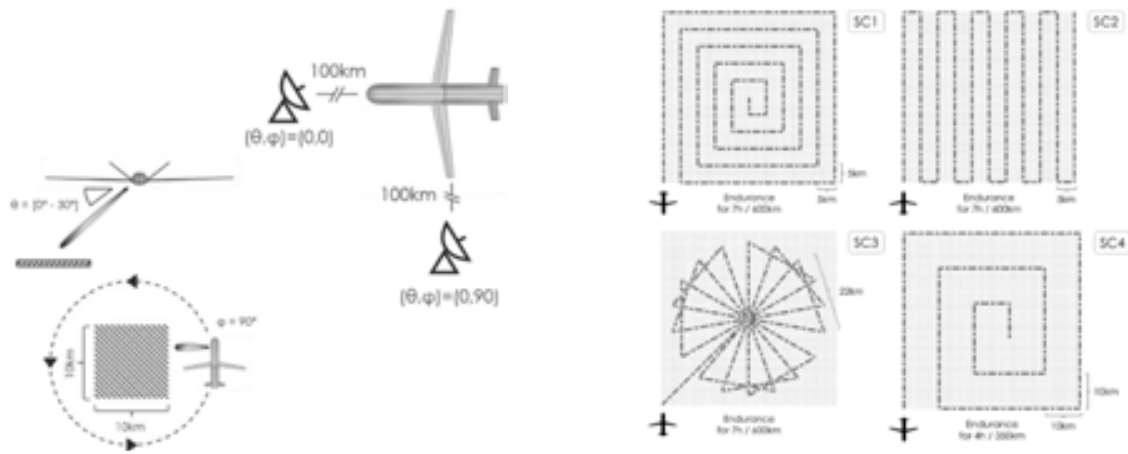

Figure 22. The simplified simulation scenario of paper III (left) and the considered trajectories in the case study of paper IV (right), adapted from papers III and IV.

To be able to include the trajectory information into the optimization, an additional and independent module that performs an initial analysis of the flight path has been specifically developed in MATLAB and included in the MDO framework of paper IV. The module divides the trajectory into discreet points that are placed $100 \mathrm{~m}$ apart, and then it exports the position and attitude data of the aircraft so that they can be used in the subsequent disciplinary simulations. More specifically, the aerodynamics, stability, trim, sensor, and radar models are evaluated at all the discretized points of the trajectory, and then the complete mission, surveillance, and stealth performance of the aircraft is calculated for the entire surveillance scenario. Compared to the simplified simulations of paper III where the angular sectors are fixed (see Figure 23 left), this new approach allows to consider numerous points in the intended operating environment, and hence, it takes into account all the potentially critical points in order to provide a more accurate representation of the performance (see Figure 23 right).
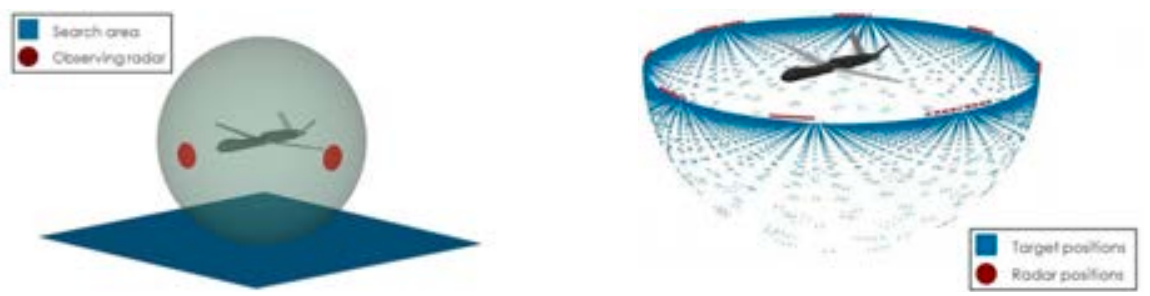

Figure 23. The radar and target positions in the simplified simulation (left) and in the trajectory analysis (right), adapted from paper IV.

\subsection{Computational Performance}

This section elaborates on the two technical solutions (metamodels and decomposition architectures) which were implemented in order to increase the 
computational performance of the proposed MDO framework. The work that is presented here is based exclusively on papers III and IV, while the aim is to investigate the strategies that enable faster optimizations of UAVs and in turn allow a better integration within the PDP (answer to RQ3).

\subsubsection{Efficient optimization strategies}

A first approach towards tackling the issue of increased computational demands is to implement an optimization architecture that can decompose the problem in a way that ensures feasibility of the solution and enables a better framework performance. The main challenge which is presented in the case studies of papers III and IV is that the optimization problem considers design variables relative to both the airframe configuration and the sensor system which means that the algorithm not only has to tackle a very large number but also a very diverse sample of parameters. On the whole, the former issue can slow the entire process since it corresponds to a more complex computational problem, while the latter can often lead to a local optimum because the algorithm might lose some sensitivity towards either the airframe or the sub-system variables.

To this end, a promising strategy that has shown significant benefits when models like the sensor system must be included in the framework is the hybrid asymmetric approach between the MDF and CO architectures (Chittick and Martins, 2008). The main principle of this multi-level methodology is that the airframe-related disciplines are put on a higher (global) level, whereas the system-related models are optimized on a lower (local) level. Here, the local optimizations are performed independently for each one of the global evaluations, and as such, each level is controlled by a specific and unique set of variables, objectives, and constraints. In this way, it is possible to reduce the total number as well as isolate the different types of parameters, and therefore, the problem can be broken into smaller parts which in turn allow to reach the final solution much faster and with much greater accuracy.

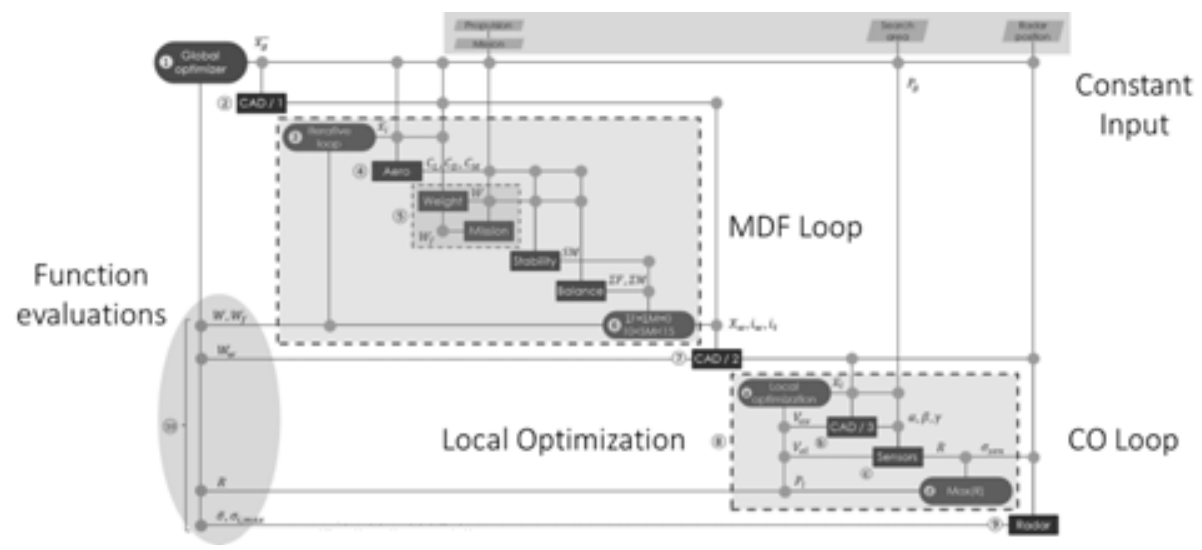

Figure 24. The multi-level asymmetric hybrid architecture, adapted from paper III. 
In paper III, a hybrid asymmetric multi-level architecture has been applied and evaluated against the traditional single-level approach where the analyses are performed sequentially and the couplings are treated as additional constraints. At the beginning of the proposed process there are several iterative loops which are used to ensure convergence of the coupled models (mission-weight, aerodynamics-stability), and then there is local optimization process where the sensor system is optimized for each airframe configuration (see Figure 24). Once the local optimization has finished, the optimum values can be either exported so that they can be assessed in the global objective function or they can be omitted from it if the design team decides that the sensor system should not be part of the optimization problem.

In total, the optimization results that are presented in paper III show that both the single- and the multi-level architectures were equally effective in the optimization of a surveillance UAV that included a sub-system model like the sensors operation. Nevertheless, there are significant differences in the time that each strategy required, and more specifically, it can be seen that the multi-level architectures were significantly faster because the global optimizer had to tackle a much simpler problem (see Figure 25). Overall, the above results are a further validation of the multi-level approach in MDO case studies that consider the aircraft on-board system models, and they indicate that this can be a very effective strategy in enabling a more broad, but yet efficient, expansion of the traditional UAV optimization frameworks.
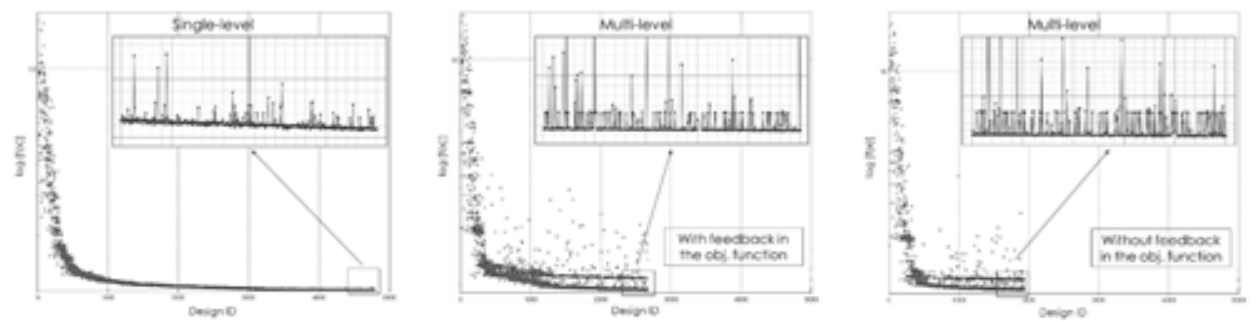

Figure 25. The convergence performance of the single-level (left) and multi-level strategies with (center) and without (right) feedback in the objective function, adapted from paper III.

\subsubsection{Applications of metamodels}

The second approach towards increasing the performance of the MDO framework which has been used in the present research is to develop and implement metamodels so that the computationally expensive disciplinary analyses can be replaced with a more suitable solution for conceptual design. The main issue is that in many cases there are no adequate low-fidelity solutions for some of the models, and hence, highfidelity tools are inevitably the only alternative which can capture the physics of the problem and close the optimization loop (Safavi, 2016). As expected, high-fidelity tools may offer very good simulation accuracy and allow for unconventional configurations to be considered, but at the same time, they also pose a significant computational 
burden which can be clearly disproportional to the rest of the models and the total level of fidelity in the framework.

In this thesis, the primary aim is to efficiently bring more knowledge of the airframe configuration and sub-system operation in conceptual design, and in this respect, different metamodeling methodologies that can fit the particular task of optimizing a surveillance UAV at a reasonable loss of accuracy have been investigated. The focus here is on the sensor and radar performance models which may be relatively fast with single calculations, but at the same time, they can become extremely computationally expensive when multiple view angles have to be evaluated. To add more to this, both models depend strongly on a detailed representation of the aircraft outer surfaces, and therefore, there is a need to update the geometry which can induce further delays especially when an independent CAD tool is used in the framework.

For the simplified surveillance scenario that is presented in paper III, only a small number of angular sectors are monitored, and therefore, the view angles are provided as constants according to the preferences of the design team. Excluding this additional factor from the inputs of the metamodel reduces significantly the complexity of the response function, and in turn enables the approximation algorithm to achieve a very low error for both the sensor and radar models. The proposed process for this type of cases can be seen Figure 26, and it is generally based on the development of one metamodel for each critical view angle by using an initial training sample which is gradually expanded until a higher and thus acceptable accuracy has been achieved.

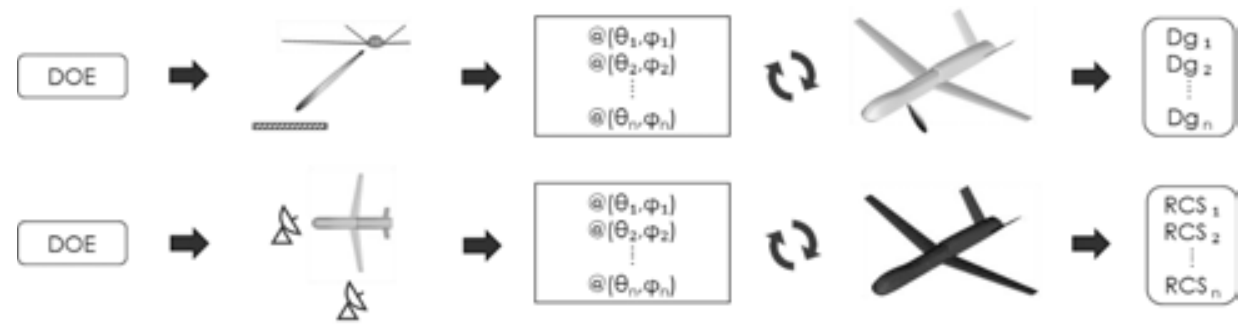

Figure 26. The proposed metamodeling methodology for cases where only a small predefined number of view angles is considered.

For the more advanced case study that is presented in paper IV, a novel metamodeling strategy was developed in order to tackle the issue of having to simulate the sensor and radar models in multiple view angles. In principle, the proposed approach is based on having one metamodel for each one of the angular sectors that comprise the hypothetical sphere around the aircraft, and in turn activate it during optimization only if the target or the ground radar is located inside it (see Figure 27). The central idea here is to generate a "look up" table inside the MDO framework which makes it possible to retrieve the performance data of the UAV at all points of the mission but without any restrictions or any need for further simulations. Overall, the applicability of this methodology is successfully tested against 4 totally different cases, 
and it is shown that it can be an efficient way to further expand the MDO framework with more applications that are position- and attitude-dependent.

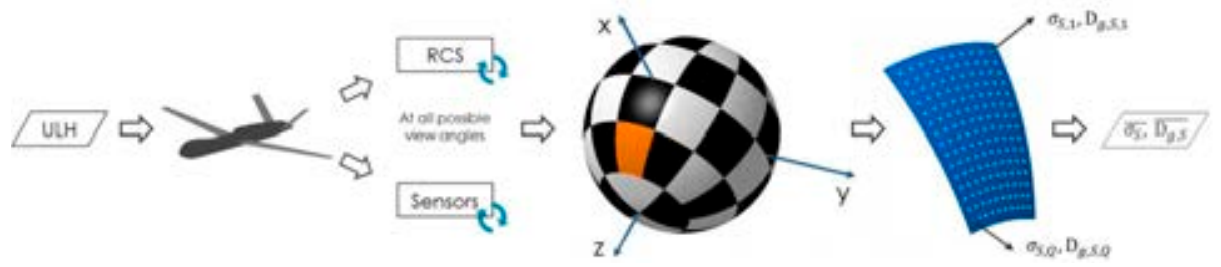

Figure 27. The proposed metamodeling methodology for capturing the response of the position- and attitude- dependent disciplines, adapted from paper IV.

\subsection{Optimization Results}

This section presents the obtained results and findings from the optimization of two exploratory UAV case studies which were elaborated in papers III and IV. In the first part, the basic MDO framework functions as well as its operation are validated, in the second part the effect of the proposed analysis capabilities in the design is assessed, and in the last part an efficient way to handle the optimization data is proposed (answers to RQ2 and RQ3).

\subsubsection{Validation of the framework}

The validation of the proposed MDO framework and the evaluation of the obtained solutions was performed in paper III by a series of optimizations which aimed to improve the capabilities of a surveillance UAV in terms of mission, sensor, and radar signature. More specifically, four different optimization runs with four different objectives were herein considered, and a visual representation of the optimum configuration is presented in Figure 28. Compared to the baseline configuration, all optimizations were able to improve the design relative to each objective, while at the same time, the validation of the optimal points by using high-fidelity simulations showed that the total metamodel error ranged from $1 \%$ to $9 \%$.

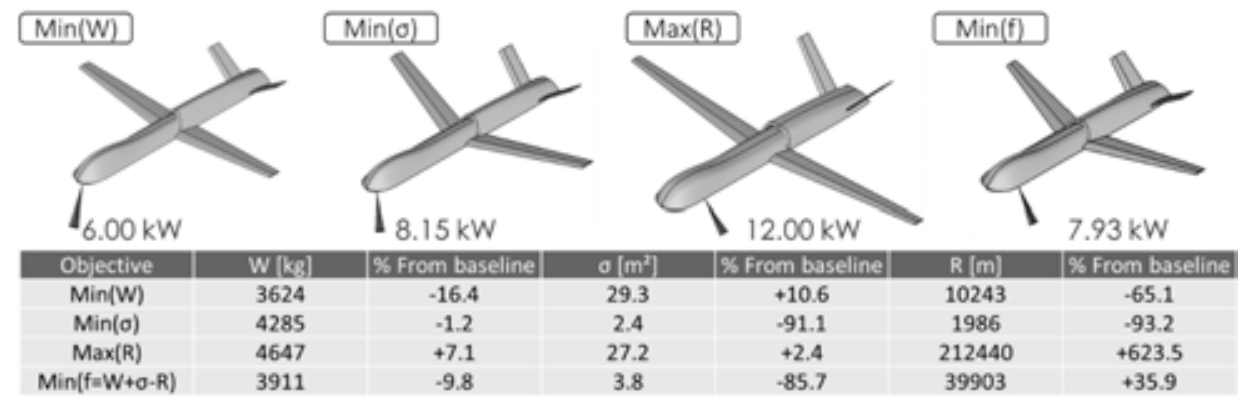

Figure 28. The optimization results from the evaluation of the framework against four different objectives, adapted from paper III. 
Apart from validation, the above results also revealed that there is a strong coupling between mission, sensor, and radar performance as seen by the values of weight $W$, radar cross section $\sigma$, and sensor effective detection range $R$. Optimizing for one objective at a time has a negative effect on the other two, while the best values that the compromise solution $f$ found were also far from the optimal points that the individual objectives were able to locate. Overall this justifies the selection of the sensor and radar performance as enhancement disciplines, and shows that further improvements to the traditional optimization framework for UAV design can be achieved if the hidden dependencies between mission, surveillance, and survivability are taken into account in MDO.

\subsubsection{Assessment of the capabilities}

The position and attitude analysis capabilities were included in the optimization by means of several different search scenarios, and they were evaluated in paper IV by exploring a concurrent mission, sensor, and radar optimization under trajectory constraints. The results which were obtained herein for each one of the evaluated search scenarios are presented in Figure 29, and it can be seen that although the objective function is the same, the optimal design configuration is quite different between each case. Here, the best detection probability was achieved in SC1 since the search pattern was able to cover a very large part of the search area, whereas the best stealth characteristics were achieved in SC3 since in this trajectory only the view angles with low RCS were exposed to the ground radars. Hence, this leads to the conclusion that the consideration of the trajectory can be clearly an essential element of MDO, and in particular, it shows that this type of information regarding the future usage of the product can be a critical aspect which should be taken into account even from the earlier stage of the process.

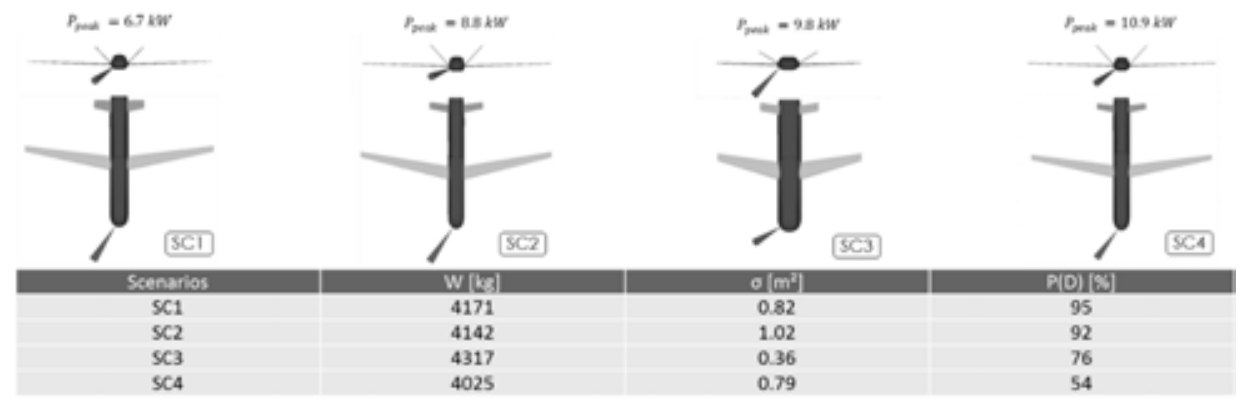

Figure 29. The optimal configuration of the UAV for each one of the four different search scenarios, adapted from paper $I V$.

In addition to the above results, a further investigation was carried out in order to determine whether or not the exclusion of the trajectory from the computations can lead to sub-optimal designs. More specifically, an optimal design was first identified by using the simplified analysis methods which were shown in paper III, and then this 
particular configuration was simulated in all the above trajectories. The obtained results showed that this seemingly optimal design was actually underperforming in all the considered scenarios, and in fact, in some cases the performance was worse than $24 \%$ compared to the cases where the trajectory constraints were included in the problem formulation. This is a further indication of the critical nature of the trajectory calculations in MDO, and it supports the initial claim that even the best UAV designs are bound to illustrate unwanted field effects if details such as the expected operating environment are omitted from the optimization.

\subsubsection{Tools for data visualization}

Given the fact that the optimization framework is functional and can generate results, the next step towards achieving organizational integration of MDO is to be able to present the findings in a way that is meaningful to the decision-making team. Thus, there is a need for improving the existing data-management methods but also a need to develop visualization tools that will allow designers to perform a better exploration the design space. A first approach towards these directions is presented in paper III, and it is based on the MATLAB GUI tool which has been used herein to develop a simple but also user-friendly environment for navigating among the identified optimal designs (see Figure 30).
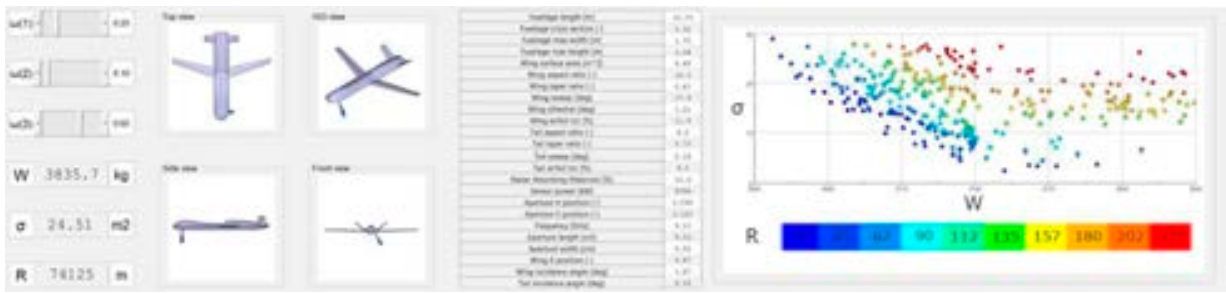

Figure 30. The first version of the data management and visualization tool, adapted from paper III.

In this first version of the GUI tool, the main functions allow the user to interactively change the weights of each attribute, and then receive the corresponding values of the objectives as well as the design variables. A visual representation by means of a "bubble" chart is also possible in order to provide a better perspective regarding the relative position of the chosen design point against the total design space. By presenting the optimization results in this way, the design team has now access to several Pareto-optimal configurations instead of only just one, and therefore, this tool can be an ideal supplement of MDO especially in conceptual design where multiple configurations need to be evaluated in a very short time. 


\section{4}

\section{Discussion and Conclusions}

In the first part of this chapter, the work that has been previously presented in this thesis is collectively summarized and discussed in light of the three main research questions. The discussions are thus divided into three topics which start by assessing the current and future possibilities in the optimization of UAVs, then followed by a description of the strategies and tools that can enhance the PDP, and finally concluding with a presentation of the methods which can lead to an improvement of the MDO capabilities. The aim of this first part is to primarily compare the current work with previous studies but also to identify and present the possible generalizations as well as limitations of the proposed methods.

In the second part of this chapter, the most interesting findings of this thesis which were based on the previously presented theory, contributions, and discussions are brought together in order to give a brief but yet clear answer to the each research question. Here, the research questions are re-stated and they are answered in a simple way which has the form of a short concluding outline that aims to provide the reader with the most important elements of this work. Finally, this chapter presents the identified directions for future work according to the gaps as well as needs of the current project, and provides several possible ideas to be used as inspiration by others in order to pursue a further research continuation.

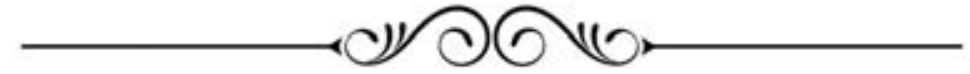




\subsection{Discussion}

In this section the research work that has been presented in this thesis is critically discussed by considering the effects of the assumptions and the limitations, while the results are further assessed by taking into account factors such as their validation and the repeatability of the method. The section is divided into three parts which focus on the current MDO possibilities (RQ1), the improvement of the UAV capabilities (RQ2), and the enhancement of the PDP (RQ3).

\subsubsection{Current practices and future possibilities}

One of the main gaps in MDO of UAVs that this thesis identified and subsequently attempted to fill is the need for expanding the traditional optimization frameworks with new and powerful features. Over the years, there have been numerous attempts to enhance the computations with more and faster models, but the main problem to this date is that several disciplines have either been overlooked or totally omitted. In its capacity to support the development process, MDO should be able to holistically assess the behavior of complex systems, and as such, the main challenge here is to concurrently but also efficiently consider further disciplinary as well as analysis capabilities.

The descriptive study which was used to identify the current technical and organizational research gaps and trends in MDO was based on two literature reviews which are presented in papers I and II respectively. A rigorous review methodology that is based on the progressive elimination of papers according to their relevance was herein implemented in order to ensure that all the important publications were taken into account. In total, the criteria which were used in this review considered only the MDO studies that are applied on general aviation aircraft and UAVs, while at the same time, a further limitation is that the examined time period has been restricted to the last decade in order to decrease the vast sample of available papers. Hence, it should become clear that the research gaps as well as the proposed roadmap for MDO are only valid in aerial vehicle design, and thus, further generalization and expansion of both the findings and the mapping process to other complex engineering products should be done with caution since it has not been verified in the current work.

Overall, the findings of the review point to the fact that the basic aeronautical disciplines like aerodynamics, propulsion, and weight estimation have been receiving a disproportional amount of focus which is explained by the fact that they are necessary in order to capture the basic mission performance of the aircraft. Nevertheless, it is often the case that the disciplinary couplings cannot be captured with only those models, and in fact, many studies have shown that adding supplementary modeling factors can lead to a better understanding of the system. In view of this, there is clearly a need to expand MDO towards the front (market and customer) and rear 
(manufacturing and testing) end of the development process, while accordingly, there is also a need to enhance the framework with more simulations and analyses that can give a better understanding of the tradeoffs between the requirements.

What is more, the literature review identified that the technical aspects of MDO have over the years received a significant amount of attention which in turn has shifted the focus away from the organizational and cultural aspects of its integration within the development process. At present, this is a major hinder towards the employment of MDO in the manufacturing industry, but also an important area where further research is required in order to align the current framework developments with the organizational need for more knowledge earlier in the PDP. To this end, an initial attempt to deal with this issue is to work towards more efficient solutions that can enable higher fidelity to be considered even in the conceptual stage, whereas at a secondary level, visualization, validation, data management, process mapping, and enhancement of the results with testing can be the next step towards a more complete MDO process.

\subsubsection{Improvement of the MDO capabilities}

To address the identified needs for a framework expansion, the present thesis has investigated the addition of two new disciplinary models (papers III and IV) as well as a further analysis capability (paper IV). The models which were chosen for this study were the performance of the sensor system as well as the radar signature of the aircraft, and the rationale is that those design aspects are instrumental in the development of surveillance UAVs but have rarely been included in MDO. Accordingly, the capability which is evaluated herein is the consideration of the search trajectory, and its importance for MDO is that this supplementary factor can increase the knowledge of how the design will perform under various customer preferences but also different usage scenarios. The effects of the new additions were evaluated by using two exploratory optimization case studies of surveillance UAVs, and it was generally shown that the proposed framework extensions have the power to drive the final design and reveal aspects that would have otherwise been hidden from the decision makers.

Starting with the disciplinary models, the results of the optimization showed that there are strong couplings between the mission, surveillance, and stealth performance, and therefore, the main advantage of the proposed additions is that they are able to provide more information about the critical disciplinary dependencies. More specifically, the radar signature and sensor models were able to provide metrics regarding the observability and search effectiveness of the aircraft, and hence, it was possible to include these values into the objective function and in turn explore a much larger design space. On the downside, the main limitation is the computational burden which can be imposed on the conceptual design framework due to the high-fidelity nature of the new tools, but nevertheless, it has also been shown in this case study that this can be tackled through the use of metamodels or efficient architectures. 
Overall, the response of the framework and in particular of the new models was verified by using available data from similar UAV applications, while in general, the development methods which were followed here can be easily adapted to further types of aircraft with the same or analogous design requirements.

As far as the analysis of the trajectory is concerned, it can be seen that this can also be a decisive factor in the design of UAVs, and more specifically, the main benefit for MDO is that it can increase the computational depth of the framework simulations by taking more operating conditions into account. Compared to the "conventional" two-dimensional way of treating the optimization of UAVs, this new approach adds more information about the position and attitude of the aircraft which is then used to enhance the calculations of the trajectory-dependent models like for example the mission, radar, and sensor performance. As expected, the multiple trajectory points are bound to require numerous analyses, and hence, the main limitation of the method is that it can be a heavy computational burden when high-fidelity tools are included in the framework. In total, the trajectory analysis can be a valuable tool during concept selection where there is a need to consider the customer preferences but also higher freedom to make decisions, while in general, the obtained results show that it can be an indispensable modeling element in both UAV and general aviation applications with similar disciplines.

On the whole, the framework that was presented herein was generally built on the concept of modularity which allows designers to change tools according to the needs and availability of each organization. To this end, it should be noted that the current tools for the mission and radar simulation are proprietary in-house codes of the project's industrial partner, however, there are several available freeware and equally powerful solutions that can easily replace them. What is more, the overall model development effort of all the new additions was performed in academia by using limited guidance from the industry, and therefore, one conclusion of this project is that it is possible to replicate this endeavor in other settings where there is partial or no help from domain experts. To sum up, the hardware which was used for the development of the models and the optimization of the design was in all cases a personal computer, and in this respect, it can be inferred that it is a realistic goal for the industry to adopt this method and enhance the development process at a minimum time as well as monetary cost.

\subsubsection{Enhancement of the development process}

As a first approach to improve the current status of MDO within the development process of aerial vehicles, a roadmap that summarizes and collectively presents the best practices was proposed (see paper I). The aim of the roadmap is to fill the gap of incomplete process mapping by combining the identified MDO elements from the literature review in a suitable workflow environment that also includes people and activities. As stated above, the applicability of the roadmap is only limited to the 
design of aerial vehicles, but at the same time, it has been built as a flexible framework that can easily undergo future changes in order to adapt itself in further design applications. On the whole, the proposed roadmap is a first approach towards a more stringent representation of MDO, and therefore, it can benefit from future expansions both in terms of technical but also organizational and cultural elements. Presently, the roadmap has only been applied in two exploratory case studies within academia which have been verified by the project's industrial partners, but nonetheless, additional work is required to evaluate it in more design scenarios and validate it against an actual UAV development case.

As a second approach towards the enhancement of the development process, this research explored and evaluated more efficient computing solutions that have the potential to reduce the computational requirements of the analyses (see papers III and IV). The performance of the framework has been traditionally the bottleneck in most optimization case studies, and as a result, this has generated a negative attitude in the manufacturing industry against MDO. Consequently, the efficient computing solutions are viewed in this context as enablers for a better organizational integration, and the reason for this is that they allow for faster optimizations which in turn are expected to assist designers in making better decisions earlier in the PDP.

Metamodels are the first efficient computing solution that is proposed by this thesis, and it is based here on the concept of bringing information from the high-fidelity tools into the conceptual design by knowingly tolerating a small approximation error. For simple scenarios where spatial information is not a requirement it was shown that simple metamodeling approaches can be adequate, however, when several points need to be simulated like in the case of a trajectory analysis then the entire response around the aircraft must be evaluated. The proposed strategy of multiple metamodels is generally able to tackle this problem at a low loss of accuracy, whereas the main limitation is that it requires a significant development time since numerous simulations need to be conducted in advance. Overall, the followed methodology is tested on the sensor performance and radar signature models, but it can easily be generalized to further disciplines that also require attitude and positon data like for example communications, noise propagation, and charging of solar panels.

Asymmetric decomposition architectures are the second efficient computing solution and their main benefit is that a computationally expensive process is treated as a local optimization in order to simplify the problem. In this case study, the problematic element was the simulation of the sensor performance which required numerous evaluations and also added supplementary variables which were only relevant to the system but not the airframe configuration. On the whole, the strategy to have a system as a local optimization has the main benefit of helping the algorithm convergence and in turn improving the speed of the optimization, but on the downside, the system design is not always the optimal since the algorithm might lose some sensitivity. Hence, asymmetric architectures can be a good alternative when on-board systems have to be 
included in the optimization, and this can easily be generalized to a number of further applications like for example the hydraulic, control, electric, and undercarriage systems.

Finally, the third approach to enhance the PDP is through the use of support methods that promote integration like for example data management and visualization tools (see paper III). The issue here is that the decision-making team is seldom interested in one design, but instead, they prefer to have knowledge over the entire design space. The benefit of the proposed management and visualization tool is that it gives designers the freedom to navigate the various designs, and subsequently it allows them to quickly explore the performance of different configurations before a decision is made. In total, the tool is generic enough to handle a wide range of optimization cases as well as non-aircraft design applications, while on the downside, the main limitation is that a certain amount of time has to be spent for the development of the functions and the integration of the optimization results.

\subsection{Conclusions}

This section collectively sums up the most important findings of the present thesis, and concludes by providing a final answer to each one of the raised research questions. In addition to this, the second part of this section includes a short summary of the complete research work and its meaning to the general MDO field, while several directions for conducting future work according to the current outlook are provided as a further motivation.

\subsubsection{Answers to the research questions}

RQ1: What are the current research gaps, trends, and improvement possibilities in $M D O$ of $U A V s$ ?

At present, the research emphasis is on improving the design through the concurrent consideration of analyses that are pertain to the engineering department, while the marketing and manufacturing aspects of the product have been typically omitted. Furthermore, the traditional MDO frameworks for UAV design have been focusing on the basic aeronautical disciplines, whereas critical case-specific requirements have been either excluded or treated through simplified simulation scenarios. Apart from this, it can be generally seen that MDO has been addressed usually at a technical level which in turn has created a gap regarding the particulars of its organizational and cultural integration within the manufacturing industry. To this end, the performance of the optimization is one way of dealing with this problem and it is still the center of attention in the majority of MDO studies, however, it can also be concluded that further additions are required in order to illustrate the benefits of MDO for both the designers and the company. In this light, it is clear that MDO should be able to work 
in parallel with the decision-makers, and hence, there is a clear need for a stringent mapping of the process but also a further and critical gap in terms of developing tools that will allow a better management of the optimization results.

RQ2: Which are the most critical additions towards enhancing the MDO of surveillance $U A V s$ ?

The main purpose of surveillance UAVs is to provide sufficient data regarding the observation of a given object within a specified search area, whereas at the same time, if their operation is over unfriendly territory, then it is necessary to remain undetected by the ground radars. As a direct consequence of this, it is necessary for the optimization process to be able to capture those new design requirements, and therefore, two critical disciplinary models that must be considered in MDO are the radar signature of the aircraft and the performance of the sensor system. Nevertheless, the aforementioned models but also the mission performance are in reality position and attitude dependent, and hence, it is necessary to analyze them in multiple points in order to get a safe estimate of their response in each scenario. This raises the need for a further trajectory analysis, and in this thesis this is treated as an input constraint which indicates what is the search pattern that will be used for detection and how the UAV is intended to fly the overall mission. Apart from the above essential functions, the trajectory analysis adds further knowledge regarding the intended future usage of the product, while at a secondary level, it is also a further strategy towards including and subsequently expressing the customer preferences and marketing possibilities in the MDO process.

RQ3: How can MDO be integrated in the manufacturing industry and support the PDP of $U A V s$ ?

One of the reasons that the manufacturing industry has been reluctant to adopt MDO is due to its performance shortcomings, and hence, in order to achieve a true integration in the PDP it is first and foremost important to be able to demonstrate better and faster results. To this end, the research that is presented here has shown two possible ways of enabling more efficient optimizations, and those are namely through the use of metamodels and multi-level decomposition architectures. In this way, it is possible to generate more simulation data from the computationally heavy, but yet critical, disciplines earlier in the process, and subsequently use this information to explore the design space and make better decisions during the stage of concept selection. Aside from the above, and in order to fill the gap of incomplete process mapping and lack of management tools, this thesis has presented a roadmap for applying MDO on UAVs as well as some preliminary work on how the optimization results can be better integrated in the PDP. Overall, the aim of roadmap is to standardize the process by acting as a checklist of the important elements and activities that must be taken into account, while the proposed data management methods aim 
to demonstrate that assessment of the design tradeoffs and visualization can be an equally crucial part of the MDO process.

\subsubsection{Outlook and future work}

Over the last years, MDO has shown great potential in improving the overall product quality and development times of UAVs by enabling more design knowledge early-on in the PDP. Nevertheless, MDO is still incomplete in terms of what is being typically modeled, while at the same time, it has never been truly integrated in the manufacturing industry. Consequently, this thesis aims to provide solutions to the aforementioned needs by expanding the traditional optimization framework and proposing further strategies that will allow MDO to be used more efficiently in the PDP. Overall, the results of this work show that the new disciplinary additions can extend the range of design requirements that can be captured by the framework, whereas further work on strategies that aim to support the use of MDO in the PDP show that this can be a promising solution towards the final acceptance by the industry.

In view of this, it becomes clear that a logical improvement direction of the MDO field is to strive for more capabilities but also more efficient and integrated processes. The former can be easily achieved by adding new disciplinary models and analysis capabilities, and to this end, some very interesting topics for future research are to include manufacturability and production aspects, test rig development models, analyses in the context of a network of systems, and lastly, supplementary market and customer inputs. As far as the process efficiency is concerned, there is further margin to improve the performance of each individual model by considering faster tools, more accurate metamodeling techniques, and smarter architectures, while in terms of integration, there is a need to expand the data management tools but also a need for a better mapping of the process with a more detailed roadmap.

Finally, a research area that is of great interest for achieving even better results in terms of design quality is the enhancement of the MDO framework of both UAVs and general aviation aircraft with data from subscale testing. In the past few years, additive manufacturing techniques and commercial of-the-shelf parts have reduced the times to develop prototypes which are now increasingly being used to retrieve information that would otherwise be impossible to know with just computer simulations. Henceforth, a research endeavor with a lot of potential is to look into the particulars of this hybrid MDO-subscale approach, and more specifically, on how this can be efficiently used with the rest of the activities of the PDP in order to provide more answers regarding the critical design aspects and the validity of the obtained optimization results. 


\section{References}

Agte, J., De Weck, O., Sobieszczanski-Sobieski, J., Arendsen, P., Morris, A., and Spieck, M. (2009), "MDO: Assessment and direction for advancement-an opinion of one international group", Structural and Multidisciplinary Optimization, Vol. 40, No. 1-6, pp. 17-33.

Allison, D., Morris, C., Schetz, J., Kapania, R., Sultan, C., Watson, L., Deaton, J., and Grandhiy, R. (2012), "A multidisciplinary design optimization framework for design studies of an effcient supersonic air vehicle", in 12th AIAA Aviation Technology, Integration and Operations (ATIO) Conference and 14th AIAA/ISSMO Multidisciplinary Analysis and Optimization Conference.

Amadori, K. (2012), Geometry-Based Design Automation - Applied to Aircraft Design and Optimization, Doctoral Thesis, Linköping University.

Amadori, K., Jouannet, C., and Krus, P. (2006), "Use of panel code modeling in a framework for aircraft concept optimization", in 11th AIAA/ISSMO Multidisciplinary Analysis and Optimization Conference.

Andersson, J. (2001), Multiobjective Optimization in Enginering Design Applications to Fluid Power Systems, Doctoral Thesis, Linköping University.

Andreasen, M. M., and Hein, L. (1987), Integrated product development, SpringerVerlag.

Austin, R. (2010), Unmanned Air Systems: UAV Design, Development and Deployment (1st Ed.), Wiley.

Balanis, C. A. (2002), Antenna Theory: Analysis and Design (3 ${ }^{\text {rd }}$ ed.), WileyInterscience.

Balesdent, M., Bérend, N., Dépincé, P., and Chriette, A. (2011). "A survey of multidisciplinary design optimization methods in launch vehicle design", Structural and Multidisciplinary Optimization, Vol. 45, No. 5, pp. 619-642.

Belie, G. (2002), "Non-Technical Barriers to Multidisciplinary Optimization in the Aerospace Industry", in 9th AIAA/ISSMO Symposium on Multidisciplinary Analysis and Optimization.

Berends, J. P. T. J., van Tooren, M., and Belo, D. N. V. (2006), "A Distributed Multi-Disciplinary Optimisation of a Blended Wing Body UAV Using a MultiAgent Task Environment", in 47th AIAA/ASME/ASCE/AHS/ASC Structures, Structural Dynamics, and Materials Conference and 14th AIAA/ASME/AHS Adaptive Structures Conference. 
Blessing, L. T. M., and Chakrabarti, A. (2009), DRM, a Design Research Methodology, Springer-Verlag.

Brezillon, J., Ronzheimer, A., Haar, D., Abu-Zurayk, M., Lummer, M., Kruger W., and Natterer, F. J. (2012), "Development and application of multi-disciplinary optimization capabilities based on high-fidelity methods," in 53rd AIAA/ASME/ASCE/AHS/ASC Structures, Structural Dynamics and Materials Conference and 20th AIAA/ASME/AHS Adaptive Structures Conference.

Cavagna, L., Ricci, S., and Travaglini, L. (2011), "NeoCASS: An integrated tool for structural sizing, aeroelastic analysis and MDO at conceptual design level", Progress in Aerospace Sciences, Vol. 47, No. 8, pp. 621-635.

Chittick, I. R., and Martins, J. R. R. A. (2008), "An asymmetric suboptimization approach to aerostructural optimization", Optimization and Engineering, Vol. 10, No. 1, pp. 133-152.

Choi, S., Alonso, J. J., Kroo, I. M., and Wintzer, M. (2008), "Multifidelity Design Optimization of Low-Boom Supersonic Jets", Journal of Aircraft, Vol. 45, No. 1, pp. 106-118.

Choi, S.-M., Nguyen, N.-V., Kim, W.-S., Lee, J.-W., Kim, S., and Byun, Y.-H. (2010), "Multidisciplinary unmanned combat air vehicle system design using multi-fidelity analysis", in 48th AIAA Aerospace Sciences Meeting Including the New Horizons Forum and Aerospace Exposition.

Cooper, R. G. (1990), "Stage-gate systems: a new tool for managing new products", Business horizons, Vol. 33, No. 3, pp. 44-54.

Cooper, R. G. (2014), "What's Next?: After Stage-Gate", Research-Technology Man., Vol. 57, No. 1, pp. 20-31.

Crawley, E., De Weck, O., Magee, C., Moses, J., Seering, W., Schindall, J., and Whitney, D. (2004), "The influence of architecture in engineering systems" (monograph).

De Weck, O., Agte, J., Sobieszczanski-Sobieski, J., Arendsen, P., Morris, A., and Spieck, M. (2007), "State-of-the-Art and Future Trends in Multidisciplinary Design Optimization", in 48th AIAA/ASME/ASCE/AHS/ASC Structures, Structural Dynamics, and Materials Conference.

Gavel, H., Ölvander, J., and Krus, P. (2008), "A quantified relationship matrix aided by optimization and probabilistic design", in 26th Congress of the International Council of the Aeronautical Sciences.

Gazaix, A., Gendre, P., Chaput, E., Blondeau, C., Carrier, G., Schmollgruber, P., Brezillon, J., and Kier, T. (2011), "Investigation of Multi-Disciplinary Optimisation for Aircraft Preliminary Design", in Aerospace Technology Conference and Exposition (SAE Technical Paper Series). 
Ghoman, S. S., Kapania, R. K., Chen, P. C., Sarhaddi, D., and Lee, D. H. (2012), "Multifidelity, Multistrategy, and Multidisciplinary Design Optimization Environment", Journal of Aircraft, Vol. 49, No. 5, pp. 1255-1270.

Giesing, J., and Barthelemy, J. F. (1998), "A summary of industry MDO applications and needs", in 7th AIAA/USAF/NASA/ISSMO Symposium on Multidisciplinary Analysis and Optimization.

Goldberg, D. (1989), Genetic Algorithms in Search, Optimization and Machine Learning (1st ed.), Addison-Wesley Longman Publishing Company.

Griffin, A., and Hauser, J. R. (1996), "Integrating R\&D and marketing: a review and analysis of the literature", Journal of product innovation management, Vol. 13 No. 3, pp. 191-215.

Haghighat, S., Martins, J. R. R. A., and Liu, H. H. T. (2012), "Aeroservoelastic Design Optimization of a Flexible Wing", Journal of Aircraft, Vol. 49, No. 2, pp. $432-443$.

Haskins, C., Forsberg, K., Krueger, M., Walden, D., and Hamelin, D. (2006), "Systems engineering handbook", International Council On Systems Engineering INCOSE.

Henderson, R. P., Martins, J. R. R. A., and Perez, R. E. (2012), "Aircraft conceptual design for optimal environmental performance", The Aeronautical Journal, Vol. 116, No. 1175, pp. 1-22.

Hurwitz, W., Donovan, S., Camberos, J., and German, B. (2012), "A Systems Engineering Approach to the Application of Multidisciplinary Design, Analysis and Optimization (MDAO) for Efficient Supersonic Air-Vehicle Exploration (ESAVE)", in 12th AIAA Aviation Technology, Integration, and Operations (ATIO) Conference and 14th AIAA/ISSMO Multidisciplinary Analysis and Optimization Conference.

Iemma, U., and Diez, M. (2006), "Optimal Conceptual Design of Aircraft Including Community Noise Prediction", in 12th AIAA/CEAS Aeroacoustics Conference (27th AIAA Aeroacoustics Conference).

Iwaniuk, A., Wiśniowski, W., and Żółtak, J. (2016), "Multi-disciplinary optimisation approach for a light turboprop aircraft-engine integration and improvement", Aircraft Engineering and Aerospace Technology, Vol. 88, No. 2, pp. 348-355.

Jeon, K.-S., Lee, J.-W., Byun, Y.-H., and Yu, Y. (2007), "Multidisciplinary UCAV system design and optimization using repetitive response surface enhancement technique", in 48th AIAA/ASME/ASCE/AHS/ASC Structures, Structural Dynamics and Materials Conference.

Karniel, A., and Reich, Y. (2011), Managing the Dynamics of New Product Development Processes, Springer-Verlag. 
Krus, P., and Andersson, J. (2003), "Simulation based optimisation for aircraft systems", in World Aviation Congress and Exposition (SAE Technical Paper Series).

Laban, M., and Herrmann, U. (2007), "Multi-Disciplinary Analysis and Optimisation Applied to Supersonic Aircraft Part 1", in 48th AIAA/ASME/ASCE/AHS/ASC Structures, Structural Dynamics, and Materials Conference.

Lee, D. S., Gonzalez, L. F., Srinivas, K., and Periaux, J. (2008), "Robust evolutionary algorithms for UAV/UCAV aerodynamic and RCS design optimisation", Computers and Fluids, Vol. 37, No. 5, pp. 547-564.

Lefebvre, T., Schmollgruber, P., Blondeau, C., and Carrier, G. (2012), "Aircraft conceptual design in a multi-level, multi-fidelity, multi-disciplinary optimization process" in 28th Congress of the International Council of the Aeronautical Sciences.

Mäkinen, R., Periaux, J., and Toivanen, J. (1999), "Multidisciplinary shape optimization in aerodynamics and electromagnetics using genetic algorithms", International Journal for Numerical Methods in Fluids, Vol. 30, No. 2, pp. 149159 .

Martins, J. R. R. A., and Lambe, A. B. (2013), "Multidisciplinary Design Optimization: A Survey of Architectures", AIAA Journal, Vol. 51, No. 9, pp. 2049-2075.

Myers, R. H., Montgomery, D. C., Anderson-Cook, C. M. (2009), Response Surface Methodology: Process and Product Optimization Using Designed Experiments (4th ed), Wiley-Interscience.

Neidhoefer, J., Ryan, J., Leahy, B., and Tripp, V. (2009), "Cooperative multidisciplinary design of integral load bearing antennas in UAVs", in 47th AIAA Aerospace Sciences Meeting including the New Horizons Forum and Exposition.

Norsell, M. (2005), "Multistage Trajectory Optimization with Radar Range Constraints", Journal of Aircraft, Vol. 42, No. 4, pp. 849-857.

Perez, R. E., Liu, H. H., and Behdinan, K. (2006), "Multidisciplinary optimization framework for control-configuration integration in aircraft conceptual design", Journal of Aircraft, Vol. 43, No. 6, pp. 1937-1948.

Persson, J. (2015), Efficient Optimization of Complex Products: A Simulation and Surrogate Model Based Approach, Doctoral Thesis, Linköping University.

Piperni, P., DeBlois, A., and Henderson, R. (2013), "Development of a Multilevel Multidisciplinary-Optimization Capability for an Industrial Environment", AIAA Journal, Vol. 51, No. 10, pp. 2335-2352.

Raymer, D. P. (2012), Aircraft design: A conceptual approach, American Institute of Aeronautics and Astronautics. 
Reuter, R. A., Iden, S., Snyder, R. D., and Allison, D. L. (2016), "An Overview of the Optimized Integrated Multidisciplinary Systems Program", in 57th AIAA/ASCE/AHS/ASC Structures, Structural Dynamics, and Materials Conference.

Rius Casals, J., Ferrando Bataller, M., and Jofre Roca, L. (1993), "GRECO: graphical electromagnetic computing for RCS prediction in real time", IEEE Antennas and Propagation Magazine, Vol. 35, No. 2, pp. 7-17.

Sadraey, M. (2008), "A Systematic Approach in Aircraft Configuration Design Optimization", in The 26th Congress of ICAS and 8th AIAA ATIO.

Safavi, E. (2016), Collaborative Multidisciplinary Design Optimization for Conceptual Design of Complex Products, Doctoral Thesis, Linköping University.

Safavi, E., Gopinath, V., Ölvander, J., and Gavel, H. (2012), "A collaborative tool for conceptual aircraft systems design", in AIAA Modeling and simulation technologies.

Safavi, E., Tarkian, M., Gavel, H., and Ölvander, J. (2015), "Collaborative multidisciplinary design optimization: A framework applied on aircraft conceptual system design", Concurrent Engineering - Research and Applications, Vol. 23, No. 3, pp. 236-249.

Savic, D. (2002), "Single-objective vs. multiobjective optimisation for integrated decision support", in 1st International Congress on Environmental Modelling and Software.

Simpson, T., and Martins, J. R. R. A. (2011), "Multidisciplinary design optimization for complex engineered systems: Report from a national science foundation workshop", Journal of Mechanical Design, Vol. 133, No. 10.

Sobieszczanski-Sobieski, J., and Haftka, R. T. (1997), "Multidisciplinary aerospace design optimization: survey of recent developments", Structural Optimization, Vol. 14, No. 1, pp. 1-23

Sobieszczanski-Sobieski, J., Morris, A., van Tooren, M. (2015), Multidisciplinary Design Optimization Supported by Knowledge Based Engineering, Wiley.

Tianyuan, H., and Xiongqing, Y. (2009), "Aerodynamic/Stealthy/Structural Multidisciplinary Design Optimization of Unmanned Combat Air Vehicle", Chinese Journal of Aeronautics, Vol. 22, No. 4, pp. 380-386.

Tice, B.P., (1991), "Unmanned aerial vehicles: The force multiplier of the 1990s", Airpower Journal, Vol. 5, No. 1, pp. 41-54.

Ulrich, K. T., and Eppinger, S. (2012), Product design and development (5th ed.), McGraw-Hill.

Valavanis, K., and Vachtsevanos, G. (2015), Handbook of Unmanned Aerial Vehicles, Springer Netherlands. 
Vandenbrande, J., Grandine, T., and Hogan, T. (2006), "The search for the perfect body: Shape Control for multidisciplinary design optimization", in 44th AIAA Aerospace Sciences Meeting and Exhibit.

Viana, F. A. C., Simpson, T. W., Balabanov, V., and Toropov, V. (2014), "Metamodeling in Multidisciplinary Design Optimization: How Far Have We Really Come?", AIAA Journal, Vol. 52, No. 4, pp. 670-690.

Volpe National Transportation Systems Center (2013), "Unmanned Aircraft System (UAS) Service Demand 2015-2035: Literature Review and Projections of Future Usage", Cambridge MA, Version 0.1.

Willcox, K., and Wakayama, S. (2003), "Simultaneous optimization of a multipleaircraft family", Journal of Aircraft, Vol. 40, No. 4, pp. 616-622.

Yan, B., Jansen, P., and Perez, R. (2012), "Multidisciplinary design optimization of airframe and trajectory considering cost and emissions", in 12th AIAA Aviation Technology, Integration and Operations (ATIO) Conference and 14th AIAA/ISSMO Multidisciplinary Analysis and Optimization Conference.

Yao, W., Chen, X., Luo, W., van Tooren, M., and Guo, J. (2011), "Review of uncertainty-based multidisciplinary design optimization methods for aerospace vehicles", Progress in Aerospace Sciences, Vol. 47, No. 6, pp. 450-479.

Zill, T., Ciampa, P. D., and Nagel, B. (2013), "A Collaborative MDO Approach for the Flexible Aircraft", in 54th AIAA/ASME/ASCE/AHS/ASC Structures, Structural Dynamics, and Materials Conference. 


\section{Appended Papers}

The papers associated with this thesis have been removed for copyright reasons. For more details about these see:

http:// urn.kb.se/ resolve?urn=urn:nbn:se:liu:diva-143672 